\title{
Market share delegation in a nonlinear duopoly with quantity competition: the role of dynamic entry barriers
}

\author{
Luca Gori* Nicolò Pecora ${ }^{\dagger} \bullet$ Mauro Sodini ${ }^{\ddagger}$
}

June 1, 2016

\begin{abstract}
This article tackles the issue of local and global dynamics in a nonlinear duopoly with quantity setting (managerial) firms and horizontal product differentiation. It studies how the dynamics of a two-dimensional discrete time map evolves by focusing on changes either in the degree of product differentiation or the managerial power in the market share bonus. By combining mathematical techniques and numerical experiments, it shows that the Nash equilibrium of the game may not describe the long-term outcomes of the market. This holds because the fixed point of the map may be unstable or because different attractors (simple or chaotic) may capture the long-term dynamics of the model. The article also analyses market dynamics when a new potential entrant tries to enter or, alternatively, a firm that was already in the market closes down and then tries to re-enter in a context where there is already an incumbent with a strictly positive quantity. The potential entrant may be subject to entry barriers or enters the market depending on the structure of consumers' preferences and the demands of products of both varieties. In particular, the article analyses some economic consequences of the non-invertibility of the map in the entry process.
\end{abstract}

Keywords: Cournot duopoly; Entry barriers; Local and global dynamics; Managerial firms; Market share delegation

JEL Classification: C61; C62; D43; L13

\section{Introduction}

Analysing problems related to the separation of ownership and control has seen a burgeoning interest in the industrial economics literature in recent decades. Since the leading article of Fama and Jensen (1983), in fact, there is an increasing attention to inquire about the reasons why the governance of competitive firms, where it is the owner's behaviour the main driving force of profit maximisation,

${ }^{*}$ L. Gori (corresponding author), Department of Political Science, University of Genoa, Piazzale E. Brignole, 3a, I-16125 Genoa (GE), Liguria, Italy; e-mail addresses: luca.gori@unige.it or dr.luca.gori@gmail.com; tel.: +39 010209 95 03; fax: +39010 2095536 .

${ }^{\dagger}$ N. Pecora, Department of Economics and Social Sciences, Catholic University, Via Emilia Parmense 84, I-29100 Piacenza (PC), Italy, e-mail: nicolo.pecora@unicatt.it, tel.: +390523599331.

${ }^{\ddagger}$ M. Sodini, Department of Economics and Management, University of Pisa, Via Cosimo Ridolfi, 10, I-56124 Pisa (PI), Italy, e-mail: mauro.sodini@unipi.it, tel.: +39050 2216 234, fax: +39050 2210603. 
is different from the governance of oligopoly firms, where it is often observed a firm's objective that does not necessarily coincides with just pure profit maximisation. In such a case, owners may hire managers and delegate output decisions to them. The delegation mechanisms are designed (generally by the owners $)^{1}$ essentially to motivate chief executive officers to achieve a competitive advantage in the market. The growth of managerial pays in large corporations has seen tremendous increases in the last twenty years (Bebchuk and Grinstein, 2005), and this fact has risen debates (both at academic and political levels) to design non-distortive kinds of incentives for the management.

The industrial organisation literature has developed a game theoretic approach to model out (the strategic use of) managerial delegation contracts in large companies. The most recognised contributions in this respect are the works of Vickers (1985), Fershtman (1985), Fershtman and Judd (1987) and Sklivas (1987), VFJS henceforth. They consider a (static) two-stage duopoly game where the owner chooses the incentive for the manager at the first stage under either a sales-based delegation contract or revenues-based delegation contract, whereas managers play a standard game in the output market (depending on whether firms are quantity setters or price setters) at the second stage. All of them share the same result, which is the cornerstone of the managerial delegation literature: in a duopoly with quantity-setting firms, profits of firm's owner who designs a contract based on the maximisation of profits and sales (or revenues) are higher than profits of the rival who just prescribes his manager to maximise pure profits. As this is a dominant strategy, the owner is induced to include incentives for sales (revenue) in the manager's contract. Therefore, the choice of delegating to external managers how to behave in the product market is the unique sub-game perfect Nash equilibrium of the managerial delegation game. ${ }^{2}$ The key result of this literature is that these incentives can strategically be used as devices to direct managers to be more aggressive in the market with the aim of increasing profits to a level higher than when firms behave just like quantity setters or price setters under standard profit maximisation.

However, equilibrium values of profits of both owners are lower than in a standard Cournot game, so that a prisoner's dilemma situation emerges. VFJS have opened the route to the study of other forms of incentive schemes for managers. We especially refer to the works of Miller and Pazgal (2002), who design an incentive scheme based on profits and relative performance evaluation, and the works of Jansen et al. (2007, 2009), who study the case of a delegation incentive for managers based on profits and market shares. Specifically, the case of market share delegation is one of the most prominent strategic commitment to non-profit maximising objectives from an empirical point of view (as is deeply discussed by Ritz, 2008). Under market share delegation players will commit to behave more aggressively in the market when products are strategic substitutes, but also that their behaviour becomes less manipulable by rivals in comparison with the cases of sales delegation and relative profit delegation. However, the prisoner's dilemma, which is a classical result of the delegation literature, still holds.

Contextually to the development of the studies mentioned above, there exists a literature led by Puu (1991) and Bischi et al. (1998) that has analysed the behaviour of nonlinear oligopoly, showing how profit maximising firms behave in the long term in repeated discrete time contexts. Since their works, nonlinear oligopoly dynamics has become a much debated topic in either cases of complete

\footnotetext{
${ }^{1}$ However, it is not uncommon to observe bargaining owners-managers in designing mechanisms of managerial pay (Bebchuk and Fried, 2004).

${ }^{2}$ See Fanti at al. (2016) for a critique on this result when the owner bargains the executive pay with his manager.
} 
information and static expectations and incomplete information with firms using specific rules to adapt their behaviour in the output market from one period to another. ${ }^{3}$ The most important results of this literature are represented by the very complicated dynamics (endogenous fluctuations and chaos) that can be observed in markets where only two firms operate in the absence of exogenous stochastic shocks. Given the importance of managerial firms in actual markets, the present article contributes to the nonlinear oligopoly literature and aims at studying how market share delegation contracts affects long-term market dynamics in a repeated duopoly where players have incomplete information. Specifically, it concentrates on local and global dynamics in a repeated discrete time duopoly with managerial firms, quantity competition and horizontal differentiation by referring to the framework developed by Bischi et al. (1998), where players have limited information and use a behavioural rule to choose the strategic variable in the product market from one period to the subsequent one. It extends the nonlinear duopoly literature developed by Fanti et al. (2012) that has studied the effects of relative profit delegation contracts in a repeated duopoly with quantitysetting firms and homogeneous products. Different from a full rationality setting, where instability of equilibria is essentially related to the existence of exogenous stochastic shocks, models that formally take into account incomplete information of agents allow scholars to introduce some behavioural rules to overcome informational lacks related to high costs of obtaining and using information efficiently (Bischi et al., 1998, 2007). In this context, instability and fluctuations are endogenous to the model in the cases of both homogeneous and heterogeneous agents.

The present article develops a mathematical analysis applied to a discrete time dynamic model by using a theoretical framework commonly used in static games in the industrial organisation literature (Jansen et al., 2007; Ritz, 2008). It also presents several numerical experiments to clarify the local and global properties of the dynamic system. In particular, the Nash equilibrium may not describe the long-term outcomes of the market. This because 1) the equilibrium may be unstable or 2) the long-term dynamics of the model may be captured by different attractors (simple or chaotic). The analysis of the dynamic system will be developed by studying of the geometric properties of the map and using simulations. In addition, given the importance (especially in a dynamic-strategic context) of the entry/exit process of firms in the market, this work presents an attempt of characterising the behaviour of a firm that is formerly out of the market and tries to enter to capture the opportunity of a positive profitability. Alternatively, we also account for the behaviour of a firm that is already in the market, then it closes down and tries to re-enter by considering the existence of an incumbent and consumers' preferences towards the new product in comparison with the existing one. In this case, market dynamics may be characterised by entry barriers for the potential entrant.

The rest of the article proceeds as follows. Section 2 sets up the (static) duopoly model with managerial firms (market share delegation) and differentiated products (introduced in the spirit of Singh and Vives, 1984), and introduces also dynamic elements into the analysis. Section 3 studies the local stability properties of discrete time dynamic system. Section 4 presents simulative exercises concentrating (separately) on the dynamic effects of changes in the degree of product differentiation and the managerial power in determining their pay. Section 5 shows market dynamics when a potential entrant wants to enter (alternatively, a firm exits and tries to re-enter) the market where there is already an incumbent and (dynamic) entry barriers may exist. Section 6 outlines the conclusions.

\footnotetext{
${ }^{3}$ See Agliari et al. (2006) for a critique of the rational expectations paradigm in economic dynamic models.
} 


\section{The model}

Consider an economy where there are two managerial firms producing in a duopolistic sector. Product of variety $i$ is produced by firm $i$, whereas product of variety $-i$ is produced by firm $-i$ ( $i=$ $1,2)$. They are perceived by (identical) consumers as horizontally differentiated products. Horizontal differentiation is strictly related to consumers' preferences. If, at equal prices, consumers do not agree on which product is the preferred one goods are said to be horizontally differentiated. This is captured by the fact that consumers differ in their preferences over product's characteristics.

Consumers. Consumers' preferences $U\left(q_{1}, q_{2}\right)$ are represented by the quadratic utility (see the work of Singh and Vives, 1984, for details):

$$
U\left(q_{1}, q_{2}\right)=q_{1}+q_{2}-\frac{1}{2}\left(q_{1}^{2}+q_{2}^{2}+2 d q_{1} q_{2}\right),
$$

where $q_{1} \geq 0$ and $q_{2} \geq 0$ are outputs of variety 1 and variety 2 , respectively, and $0 \leq d \leq 1$ is the degree of horizontal product substitutability as perceived by consumers (when $d=0$ products are actually two distinct goods and each firm behaves as if it were a monopolist in its own market; when $d=1$ products are perfect substitutes or homogeneous). In general, it is possible to account for product complementarity when $-1 \leq d \leq 0$. However, there are several phenomena that should be analysed and discussed just focusing on case $0 \leq d \leq 1$, so that in this article we concentrate only on product substitutability (which is also the most relevant case from an empirical point of view). From the utility function in (1) one can easily gets the system of inverse demands of products of both varieties, that is:

$$
p_{1}=1-q_{1}-d q_{2} \quad \text { and } \quad p_{2}=1-q_{2}-d q_{1} .
$$

In order to guarantee that prices $p_{1}$ and $p_{2}$ are non-negative for any $d \in(0,1]$, we take quantities $q_{i}$ and $q_{-i}$ belonging to set

$$
A:=\left\{q_{i}, q_{-i}: q_{i}+d q_{-i} \leq 1\right\}
$$

Firms. The technology of firm $i(i=1,2)$ has constant marginal returns to labour. This implies that the production function is $q_{i}=L_{i}$, where $L_{i}$ is the labor force hired in the firm. Average and marginal costs are identical for both firms and they are given by $0 \leq w<1$, which can be interpreted as the wage per unit of labour. Therefore, firms $i$ 's cost function is $c_{i}=w q_{i}$. As firm $i$ is a managerial firm whose aim is not just profit maximisation, we assume that the owner of each firm hires a manager and delegates output decisions to him. Specifically, each manager receives a fixed salary (which is set to zero without loss of generality) plus a bonus offered in contract which is publicly observable ${ }^{4}$ and based on market share $\frac{q_{i}}{q_{i}+q_{-i}}$ (Jansen et al., 2007; Ritz, 2008), with $i=1$, 2, where $q_{i}+q_{-i}$ is total supply. The utility of the manager hired by the owner of firm $i$ is then given by:

$$
W_{i}=\Pi_{i}+b_{i} \frac{q_{i}}{q_{i}+q_{-i}},
$$

\footnotetext{
${ }^{4} \mathrm{~A}$ contract that is publicly available implies that firms know the kind of contract agreed upon with the manager as well as the type of manager behavior. In contrast, under private information, there should be problems of signaling and uncertainty related to the available information.
} 
Eq. (4) represents the manager's utility function, where $\Pi_{i}=\left(p_{i}-w\right) q_{i}$ are the profits of the $i$-th firm and $b_{i}>0$ is the delegation variable of manager hired in firm $i$, which is usually considered as a control variable in the industrial economics literature. It is chosen in the contract-stage of the game (see VFJS), and the equilibrium values of it depends on the other parameters of the problem (e.g., production costs, degree of product differentiation, etc.). In fact, in a static managerial game where players have perfect knowledge, owners strategically choose the nature of the bonus at the contract stage (i.e., pure profits or a specific delegation contract), whereas managers choose either the quantity (in the case of quantity setting behaviour) or the price (in the case of price-setting behaviour) at the market stage. Definitely, the game is solved by using the standard backward logic. In this work, however, we consider $b_{i}$ as an exogenously given parameter and look at the dynamic properties of the model when $b_{i}$ changes. This hypothesis follows Fanti et al. (2012). Indeed, in a dynamic context such as the present one, players do not have perfect knowledge. Then they do compute neither their own best replies (given the choices of the rival) not the optimal value of weight $b_{i}$ in the managerial pay. Therefore, given the assumption of limited information, it would be of importance for players (managers) to know the direction of the change in output given $b_{i}$ and the other parameters.

Knowing that $\Pi_{i}=\left(p_{i}-w\right) q_{i}$ and using (2), the utility function of manager (4) may be rewritten as:

$$
W_{i}=\left(1-q_{i}-d q_{-i}-w\right) q_{i}+b_{i} \frac{q_{i}}{q_{i}+q_{-i}} i=1,2 .
$$

From (5), the marginal utility of manager $i=1,2$ reads as follows:

$$
\frac{\partial W_{i}}{\partial q_{i}}=1-2 q_{i}-d q_{-i}-w+b_{i} \frac{q_{-i}}{\left(q_{i}+q_{-i}\right)^{2}} i=1,2 .
$$

Dynamic setting. Consider now a dynamic setting where time is indexed by $t \in \mathbb{Z}_{+}$. By following the tradition of Bischi et al. (1998), we assume that both players have limited information and follow an adjustment mechanism based on local estimates of the marginal bonus to overcome this informational lack. In this context, managers do not know the optimal value of weight $b_{i}$, chosen by the owner of the firm as well as the market demand. Therefore, the behavioural rule used by manager of firm $i$ is given by

$$
q_{i}(t+1)=q_{i}(t)+\alpha q_{i}(t) \frac{\partial W_{i}\left(q_{i}(t), q_{-i}(t)\right)}{\partial q_{i}(t)},
$$

where $\alpha>0$ and $\partial W_{i}\left(q_{i}(t), q_{-i}(t)\right) / \partial q_{i}(t)$ is determined by (6). By taking into account (7) and setting $b_{1}=b_{2}=b$, the two-dimensional system that characterises the dynamics of this Cournot duopoly can be written as follows:

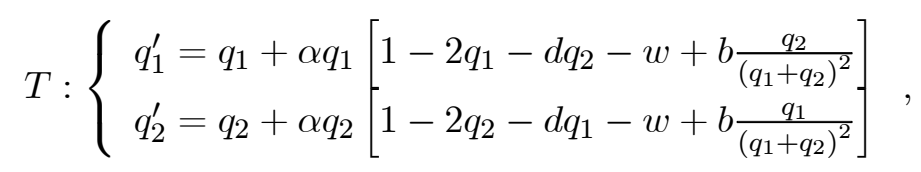

where ' denotes the unit-time advancement operator. In actual markets, there may exist various types of managers. However, in this game-theoretic set-up the owner makes a take-it-or-leave-it offer to the manager, which in turn competes in the output market with the manager hired by the rival. Then, the assumption of equal manager's attitude $\left(b_{1}=b_{2}=b\right)$ implies that players (managers) 
are homogeneous in this dynamic context. This assumption is in line with the managerial delegation literature, where the equality between the weights in the managerial contracts follows the development of the models. In addition, as we will show later in this article, the strategic interactions between homogeneous managers may lead to heterogenous behaviours.

Map $T$ is a two-dimensional dynamic system, whose iteration defines the time evolution of the quantity of products of each variety. Although the underlying theoretical economic paradigm adopted in this article (i.e., a quantity-competition duopoly with product differentiation and managerial firms with market share delegation) is similar to the price competition model studied by Fanti et al. (2014), the resulting maps have noteworthy differences from a dynamic point of view, thus deserving separate analyses.

\section{Invariant sets and local stability properties}

Map (8) is characterised by the existence of three (economically feasible) fixed points: $E_{1}=\left(\frac{1-w}{2}, 0\right)$ and $E_{2}=\left(0, \frac{1-w}{2}\right)$ located on the invariant coordinate axes, and

$$
E^{*}=\left(q^{*}, q^{*}\right),
$$

where $q^{*}:=\frac{1-w+\sqrt{b(2+d)+(1-w)^{2}}}{2(2+d)}>0$, representing the unique Nash equilibrium of the game. In addition, a fourth (economically unfeasible) fixed point of the map exists

$$
E_{3}=(\bar{q}, \bar{q})
$$

where $\bar{q}:=\frac{1-w-\sqrt{b(2+d)+(1-w)^{2}}}{2(2+d)}<0$ is conjugate to the repelling fixed point 0 of the logistic map (see (12) below). Thus, $E_{3}$ is a repelling equilibrium. Fixed points $E_{1}$ and $E_{2}$ imply that at least one firm does not produce and exits the market. One important characteristic of map (8) is that the diagonal $\Delta=\left\{\left(q_{1}, q_{2}\right): q_{1}=q_{2}\right\}$ is an invariant manifold, that is $T(\Delta) \subseteq \Delta$. In fact, map $T$ has a symmetric form, so that it does not change if variables $q_{1}$ and $q_{2}$ are exchanged, that is $T \circ S=S \circ T$, where $S:\left(q_{1}, q_{2}\right) \rightarrow\left(q_{2}, q_{1}\right)$ is the reflexion through the diagonal $\Delta$. This implies that by starting with the same initial condition $q_{1}(0)=q_{2}(0)$, the dynamics lie on $\Delta$ for every $t$. In this case, the behaviour of the dynamic system is described by the restriction of map $T$ on $\Delta$ and synchronised trajectories (i.e. $q_{1}(t)=q_{2}(t)$ for every $t$ ) are governed by $T_{\Delta}: \Delta \rightarrow \Delta$, where

$$
T_{\Delta}: q^{\prime}=f(q)=-\alpha(2+d) q^{2}+(1+\alpha-\alpha w) q+\frac{1}{4} \alpha b .
$$

Moreover, the map in (11) is topologically conjugate to the logistic map

$$
z^{\prime}=\mu z(1-z)=g(z),
$$

with parameter

$$
\mu=1+\alpha \sqrt{b(2+d)+(1-w)^{2}},
$$

through the homeomorphism

$$
h(z)=u z+v=\frac{1}{\alpha(2+d)}\left(1+\alpha \sqrt{b(2+d)+(1-w)^{2}}\right) z+\frac{\left(1-w-\sqrt{b(2+d)+(1-w)^{2}}\right)}{2(2+d)} .
$$


Thus, the dynamic behaviour of the restriction of $T$ to the invariant manifold $\Delta$, where synchronised dynamics of the identical players take place, can be obtained from the well-known behaviour of the logistic map by a homeomorphism. The topological conjugacy between maps (11) and (12) implies that the dynamics of (11) are completely known, as these can be obtained from those of the logistic map (12).

Now, we briefly recall some properties of the logistic map. The positive fixed point $z^{*}=1-\frac{1}{\mu}$ is stable for $1<\mu<3$, corresponding to $d<\frac{1}{b}\left(\frac{4}{\alpha^{2}}-(1-w)^{2}\right)-2$. Of course, the positive fixed point of (12) on the diagonal coincides with the Nash equilibrium of the duopoly game, as given by (9). At $\mu=\mu_{0}=3$ the fixed point $z^{*}$ loses stability through a flip bifurcation and for $3<\mu<1+\sqrt{6}$ a two-period attracting cycle is the unique attractor of map (12). In addition, this cycle undergoes a flip bifurcation at $\mu=1+\sqrt{6}$ causing the appearance of a four-period attracting cycle, which is followed by the well-known cascade of period doubling bifurcations leading to the chaotic behaviour of (12). In this way the behaviour of synchronised trajectories is completely known.

We note also that $q_{1}$-axis and $q_{2}$-axis are forward invariant. The map restricted on $q_{i}$-axis is the following:

$$
\left.T\right|_{q_{-i}=0}=-2 \alpha q_{i}^{2}+q_{i}(1+\alpha-\alpha w),
$$

which is topologically conjugate to the logistic map through the change of variable $q_{i}=\frac{1+\alpha-\alpha w}{2 \alpha} z$ and $\mu=1+\alpha-\alpha w$. This implies that for $\alpha \in\left(0, \frac{3}{1-w}\right]$ we obtain bounded trajectories along the invariant axis $q_{i}$ provided that the initial value belongs to $\omega_{i}=\left[O, O_{i}^{-1}\right]$, where $O:=(0,0)$ is the origin, $O_{i}^{-1}$ is the rank-1 preimage of the origin on the corresponding axis and the non-null coordinate of $O_{i}^{-1}$ is $\frac{1+\alpha-\alpha w}{2 \alpha}$. We will see later in this article that these segments will play a relevant role in the definition of the basins of attraction.

In what follows, we will consider producers starting from different initial conditions to look at whether they evolve towards synchronization, so that their dynamic behaviour is completely determined by the one-dimensional attractors of the restriction of $T$ along the diagonal $\Delta$, or (alternatively) they converge somewhere else. The local stability analysis of equilibria can be carried out by considering the Jacobian matrix associated with map $T$. It is given by

$J=\left(\begin{array}{cc}1+\alpha\left(1-2 q_{1}-d q_{2}-w+\frac{b q_{2}}{\left(q_{1}+q_{2}\right)^{2}}\right)-2 \alpha q_{1}\left(1+\frac{b q_{2}}{\left(q_{1}+q_{2}\right)^{3}}\right) & \alpha q_{1}\left(-d+\frac{b}{\left(q_{1}+q_{2}\right)^{2}}-\frac{2 b q_{2}}{\left(q_{1}+q_{2}\right)^{3}}\right) \\ \alpha q_{2}\left(-d+\frac{b}{\left(q_{1}+q_{2}\right)^{2}}-\frac{2 b q_{1}}{\left(q_{1}+q_{2}\right)^{3}}\right) & 1+\alpha\left(1-2 q_{2}-d q_{1}-w+\frac{b q_{1}}{\left(q_{1}+q_{2}\right)^{2}}\right)-2 \alpha q_{2}\left(1+\frac{b q_{1}}{\left(q_{1}+q_{2}\right)^{3}}\right)\end{array}\right)$,

By evaluating the Jacobian matrix at $E_{1}$ and $E_{2}$ we find that both fixed points are never locally stable. More precisely, we can conclude that $E_{1}$ and $E_{2}$ are saddle points when $\alpha<\frac{2}{1-w}$, whereas they are source when $\alpha>\frac{2}{1-w}$. We note also that at point $O$ the map is neither defined nor it can be extended continuously. By the study on the restriction of map (8) on both the diagonal and invariant axes we can conclude that $O$ is not locally stable.

Now, by starting from some non-negative initial production strategies, we can observe that the map in (8) is not invertible. This implies that is an iteration of (8) uniquely defines the forward trajectory, whereas a backward iteration of (8) is not uniquely defined because a point $\left(q_{1}^{\prime}, q_{2}^{\prime}\right)$ of the plane may have more than one preimage (see Mira et al., 1996 for a description of the main properties 
of non-invertible maps). More precisely, rank-1 preimages of a given point $\left(q_{1}^{\prime}, q_{2}^{\prime}\right)$ are solutions of system

$$
\left(q_{1}^{\prime}, q_{2}^{\prime}\right)=T\left(q_{1}, q_{2}\right)
$$

and the corresponding two fourth degree algebraic equations may have four or two real solutions or no real solution at all. Consequently, $T$ is of $Z_{4}-Z_{2}-Z_{0}$ type, that is we can divide the plane into regions $Z_{4}, Z_{2}$ and $Z_{0}$ according to the number of preimages (where the subscript in $Z$ denotes their number), as Figure 1(b) displays. As a direct consequence if we let $\left(q_{1}^{\prime}, q_{2}^{\prime}\right)$ vary in $\mathbb{R}^{2}$, the number of rank-1 preimages changes as point $\left(q_{1}^{\prime}, q_{2}^{\prime}\right)$ crosses the boundary that separates such regions. These boundaries are generally characterised by the existence of two coincident preimages. In this respect, we follow the notation introduced by Mira et al. (1996) and use the critical curves technique. The critical curve of rank-1, denoted by $L C$, is defined as the locus of point with two (or more) coincident rank-1 preimages located on a set called $L C_{-1}$. In particular, $L C_{-1}$ belongs to the locus of point where the determinant of the Jacobian matrix associated with map $T$ vanishes, i.e. $L C_{-1} \subseteq\left\{\left(q_{1}, q_{2}\right) \in \mathbb{R}_{+}^{2}: \operatorname{Det}(J(T))=0\right\}$ and $L C$ is the rank-1 image of $L C_{-1}$ under $T$, i.e. $L C=T\left(L C_{-1}\right)$. Unfortunately, it is not possible to obtain a solution of $\operatorname{Det}(J(T))=0$ in closed form and then we proceed through numerical simulations. Once the locus of points that defines $L C_{-1}$ (see Figure 1(a) for a graphical representation) is identified, we can obtain segments of critical curves of increasing rank and define a trapping region in the phase plane.

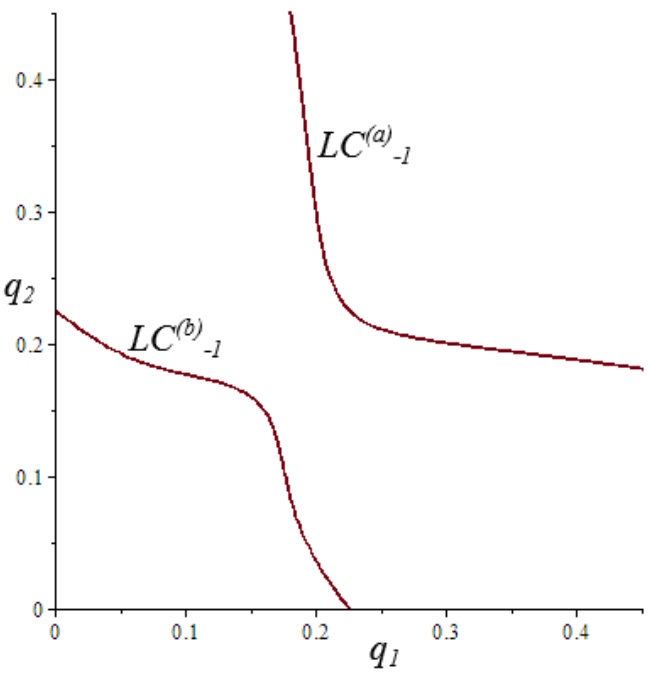

(a)

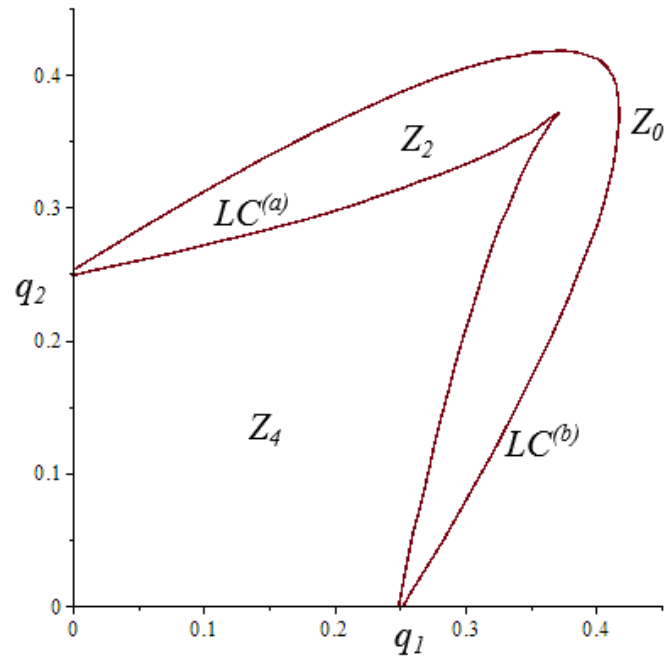

(b)

Figure 1. (a) Critical curves of rank-0, $L C_{-1}$, for map $T$. (b) Critical curves of rank-1, $L C=$ $T\left(L C_{-1}\right)$. These curves separate the phase plane into regions $Z_{4}, Z_{2}$ and $Z_{0}$ whose points have a different number of preimages. Parameter values are: $\alpha=2.48, w=0.5, b=0.375$ and $d=0.9$.

It is important to note that the local analysis around the diagonal is strictly related to synchronisation (on-off intermittency). In fact, since $T$ is symmetric with respect to $\Delta$, then its Jacobian 
matrix evaluated at a point on the diagonal $\Delta$ is of the kind

$$
J_{\Delta}(q, q)=\left(\begin{array}{ll}
J_{1}(q) & J_{2}(q) \\
J_{2}(q) & J_{1}(q)
\end{array}\right)
$$

where

$$
J_{1}(q)=\alpha q\left(-\frac{b}{4 q^{2}}-2\right)+\alpha\left(-w-2 q-d q+\frac{b}{4 q}+1\right)+1
$$

and

$$
J_{2}(q)=-\alpha d q .
$$

Consequently, the eigenvalues of $J_{\Delta}$ are both real and they are given by

$$
\begin{aligned}
\lambda_{\|} & =J_{1}(q)+J_{2}(q)=\alpha-\alpha w-4 \alpha q-2 \alpha d q+1 \text { with eigenvector } \mathbf{r}_{\|}=(1,1), \\
\lambda_{\perp} & =J_{1}(q)-J_{2}(q)=\alpha-\alpha w-4 \alpha q+1 \text { with eigenvector } \mathbf{r}_{\perp}=(1,-1) .
\end{aligned}
$$

From the hypothesis on the sign of $d$, it is worth to note that for $q>0$ we have that $\lambda_{\|}<\lambda_{\perp}$. Of course, the eigenvalue $\lambda_{\|}$, related to the invariant manifold along $\Delta$, coincides with the eigenvalue of the restriction of $T$ to $\Delta$, as is given by (11). By specialising such a result for the fixed point we get

$$
\lambda_{\|}\left(E^{*}\right)=1-\alpha \sqrt{\left.(2+d) b+(1-w)^{2}\right)},
$$

which is always lower than 1 . Moreover, the eigenvector related to the other eigenvalue is always orthogonal to $\Delta$ and independent of $q$. As we have assumed $0 \leq d \leq 1$, in what follows we focus our attention on the transverse stability of the invariant sets located on the line $\Delta$. For the fixed point $E^{*}$ the transverse eigenvalue is

$$
\lambda_{\perp}\left(E^{*}\right)=\frac{2+(1+(1-w) \alpha) d-2 \alpha \sqrt{(2+d) b+(1-w)^{2}}}{2+d},
$$

which is transversely attracting for all parameters that give bounded dynamics on $\Delta$. In particular, given the sorting of the two eigenvalues depending on the sign of parameter $d$, the stability of the Nash equilibrium $E^{*}$ requires $\lambda_{\perp}\left(E^{*}\right)<1$ and $\lambda_{\|}\left(E^{*}\right)>-1$, which reads as

$$
d<\max \left\{\frac{4-\alpha^{2}(1-w)^{2}-2 \alpha^{2} b}{\alpha^{2} b}, 2 \frac{(1-w)^{2}+2 b}{(1-w)^{2}}\right\} .
$$

Now from $\lambda_{\|}$and $\lambda_{\perp}$ we can study the local stability of cycles that can occur on $\Delta$. For a $k$-cycle $\left\{\left(x_{1}, x_{1}\right),\left(x_{2}, x_{2}\right), \ldots,\left(x_{k}, x_{k}\right)\right\}$ embedded into the invariant line $\Delta$ where synchronized dynamics take place, the two multipliers are

$$
\begin{aligned}
& \lambda_{\|}^{(k)}=\prod_{i=1}^{k}\left(J_{1}\left(x_{i}\right)+J_{2}\left(x_{i}\right)\right)=\prod_{i=1}^{k}\left(\alpha-\alpha w-4 \alpha x_{i}-2 \alpha d x_{i}+1\right), \\
& \lambda_{\perp}^{(k)}=\prod_{i=1}^{k}\left(J_{1}\left(x_{i}\right)-J_{2}\left(x_{i}\right)\right)=\prod_{i=1}^{k}\left(\alpha-\alpha w-4 \alpha x_{i}+1\right) .
\end{aligned}
$$


Also for the cycles, the conditions for stability and local bifurcations along $\Delta$ are the same as for the corresponding cycle of the quadratic map (12).

By considering a more complex situation, an attractor $A$ on $\Delta$ is an asymptotically stable attractor of map $T$ if and only if all trajectories belonging to $A$ are transversely attracting. In order to study its transverse stability, we follow the procedure adopted in Bischi et al. (1998), Bischi and Gardini (2000) and Agliari and Bignami (2010), based on the use of the transverse Lyapunov exponent

$$
L_{\perp}=\lim _{N \rightarrow \infty} \frac{1}{N} \sum_{n=0}^{N} \ln \left|\lambda_{\perp}\left(x_{n}\right)\right|,
$$

where $x_{0} \in A$ and $\left\{x_{n}\right\}$ is a generic trajectory generated by map $T$ restricted on the diagonal $\Delta$. According to the initial conditions, a spectrum of Lyapunov exponents can be defined as

$$
L_{\perp}^{\min } \leq \ldots \leq L_{\perp}^{n a t} \leq \ldots \leq L_{\perp}^{\max }
$$

where $L_{\perp}^{\text {nat }}$ is computed for a typical trajectory taken in the chaotic attractor $A$. By following Bischi et al. (1998), we recall that if $L_{\perp}^{\max }<0$, then $A$ is asymptotically stable in the Lyapunov sense, while if $L_{\perp}^{\text {nat }}<0$ and $L_{\perp}^{\max }>0$ then $A$ is no longer Lyapunov stable but it attracts a large set (the basin of attraction has positive Lebesgue measure) of points in the two-dimensional phase space. In this case, $A$ is said to be a Milnor attractor.

We recall that a closed invariant set $A$ is said to be a weak attractor in Milnor sense if its stable set has positive Lebesgue measure. It is also noteworthy that if $L_{\perp}^{\text {nat }}>0$, set $A$ becomes a chaotic saddle and trajectories that start from an initial conditions close to the diagonal can either be captured by an attractor that envelops the saddle or captured by other attractors.

\section{Numerical analysis}

In this section, we consider the situation in which firms start from different initial states. Specifically, we investigate which is the long-term outcome of the model in the case in which: both firms synchronise their actions (that is, they behave in the same way); the dynamic interaction between the two firms generates heterogeneous behaviours; there exists phenomena of path dependency, that is initial conditions matter (multistability). As we are mainly interested in the role played by parameters $d$ and $b$, in what follows we study separately how changes in the degree of product differentiation and in the weight of managerial power affect dynamic outcomes.

\subsection{The role of product differentiation in the dynamics}

We begin the analysis by considering first a parameter set in which we vary the degree of product differentiation $d$, by keeping the other parameters fixed at $\alpha=2.48, w=0.5$ and $b=0.375$. The study is first conducted by fixing the product differentiation parameter at $d=0.38$. In this case, there exists a two-piece chaotic attractor along the diagonal. In particular, Figure 2(a) depicts its basin of attraction (plotted in gray), that is the set of initial conditions in the positive orthant generating positive trajectories for every iterate and that are attracted by the chaotic attractor. In fact, it is interesting to note that different from several nonlinear duopoly models developed in the literature, it 
is possible to have trajectories that temporarily exit the first quadrant and in turn re-enter in it to be eventually captured by the attractors of the system that lie on it (see Figure 2(b)). Of course, such trajectories do not have economic sense as the quantity produced by both firms cannot take negative values. For this reason, the white region in Figure 2(a) and in all the basins of attraction depicted in the figures of Section 4 except Figure 2(b) describes trajectories in which at least one of the iterates has a negative component. In any case, in order to understand the peculiar structure of the basin of attraction of $E^{*}$ characterised by the existence of lobes in Figure 2(b), we note that 1) there exists a set $M_{1}:=\left\{\left(q_{1}, q_{2}\right) \in \mathbb{R}^{2}: q_{1}+q_{2}=0\right\}$ on which map $T$ is not defined and, in particular, there exists a focal point $O$ (see Bischi et al., 2003, for details), and 2) fixed point $E_{3}$ and its pre-images are involved in the definition of such new portions of the basins of attraction. However, such a point does not play any role if we concentrate on the map defined on $\mathbb{R}_{+}^{2}$. In fact, by introducing polar coordinates it is simple to verify that for any sequence converging towards $O$ we obtain that the image through $T$ of such a sequence converges towards a point $P \in M_{2}:=\left\{\left(\frac{\alpha b \sin (2 \theta)}{2(1+\sin (2 \theta))}, \frac{\alpha b \sin (2 \theta)}{2(1+\sin (2 \theta))}\right), \theta \in\left[0, \frac{\pi}{2}\right]\right\}$. This implies that $O$ is not an attractor of the system in $\mathbb{R}_{+}^{2}$ and any point close to $O$ (at least in our numerical settings) is mapped into a point that belongs to one of the basins of attraction of the finite distance attractors.

By focusing now on the $\omega$-limit set depicted in Figures 2(a) and 2(b), we note that such a set is an attractor in the Milnor sense. This means that several cycles embedded in the diagonal are transversely unstable. This implies that trajectories approaching to the diagonal are burst away by transversely unstable cycles. Indeed, the convergence towards the unique chaotic (Milnor) attractor of the system embedded in the diagonal occurs only after a very long transient, as Figure 2(c) displays. 


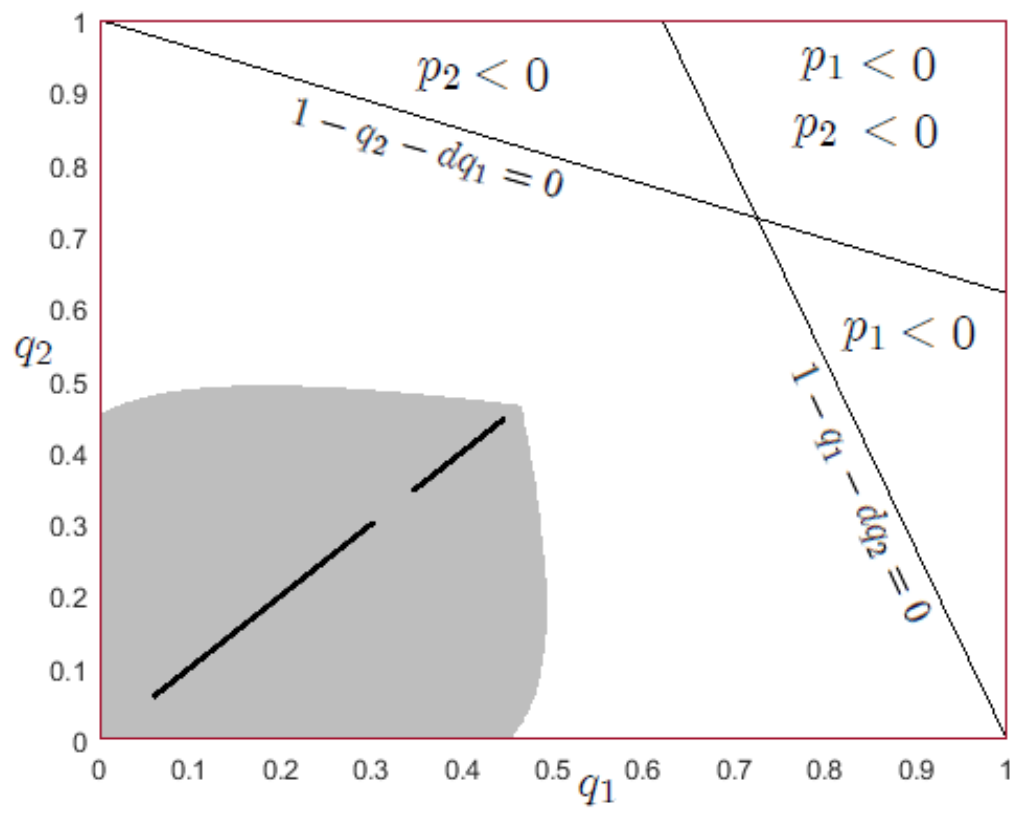

(a)

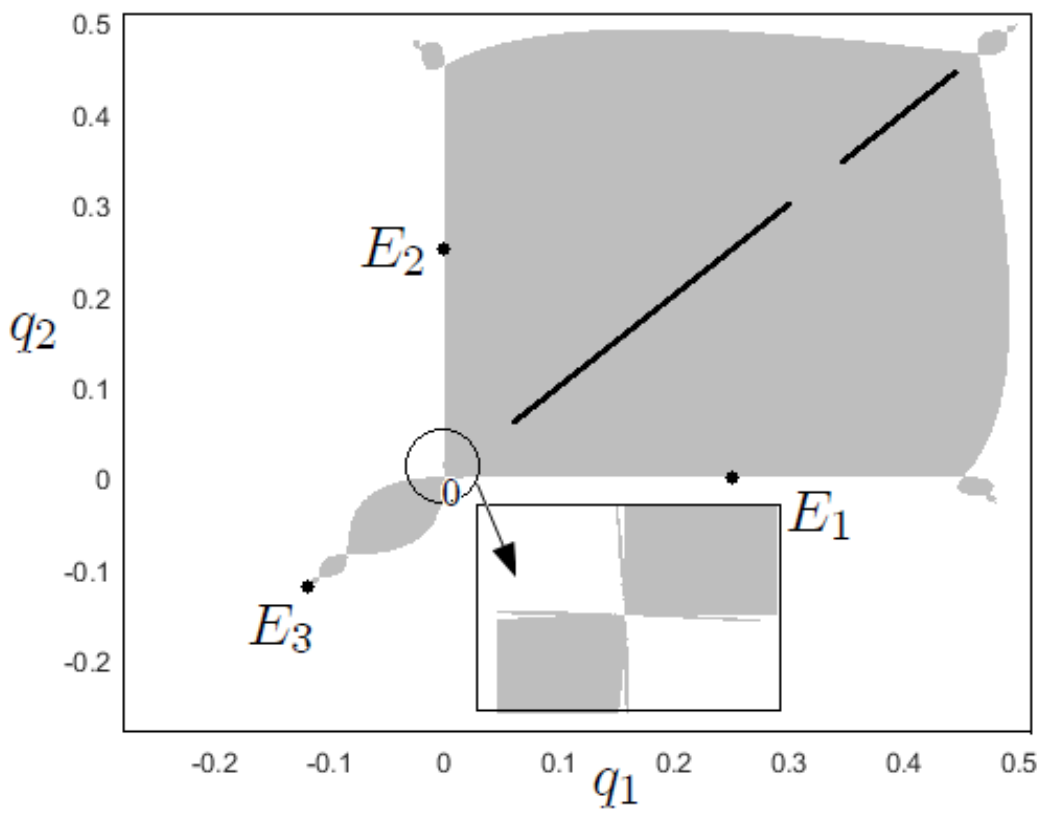

(b)

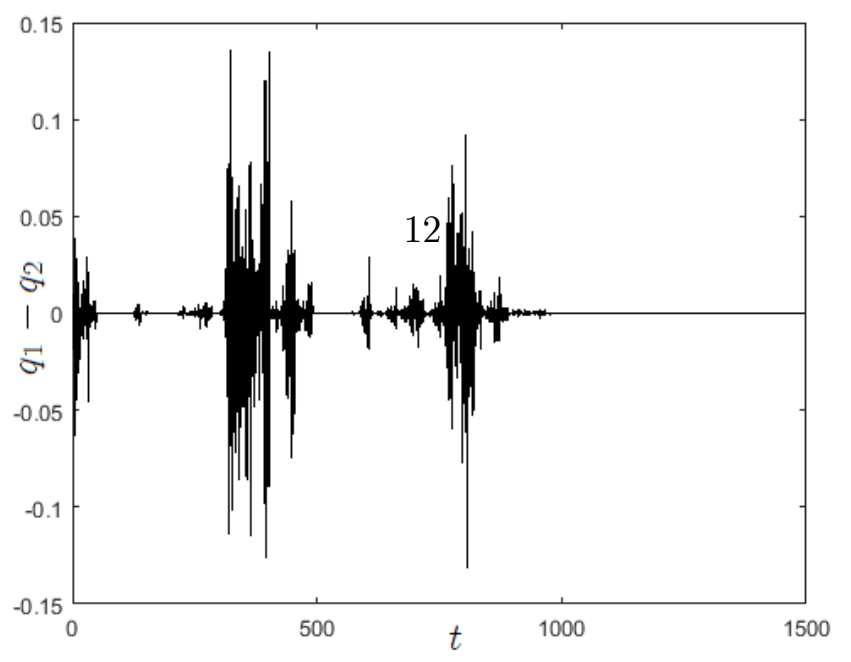


Figure 2. (a) A two-piece Milnor attractor along the diagonal. The lines with negative slopes in $\left(q_{1}, q_{2}\right)$ plane bound the space of quantities such that prices of products 1 and 2 are strictly positive. (b) Basin of attraction when non-negative constraints are not introduced. The enlargement view emphasises the birth of lobes in the basins of attraction of the Milnor attractor. These lobes are associated with the existence of a focal point, the origin $O . E_{1}$ and $E_{2}$ are fixed points located on the invariant coordinate axes and $E_{3}$ is the economically unfeasible fixed point. (c) Synchronisation occurs only after a long transient (on-off intermittency). Parameter values: $\alpha=2.48, w=0.5$, $b=0.375$ and $d=0.38$.

However, as $d$ decreases we observe an important change in long-term outcomes: trajectories starting outside the diagonal do not synchronise and are no more confined along the diagonal. Indeed, as Figure 3(a) clearly shows, a two-piece chaotic attractor that governs long-term dynamics now exists. Moreover, Figure 3(b) depicts the boundary of the chaotic attractor by the use of the critical lines technique. 


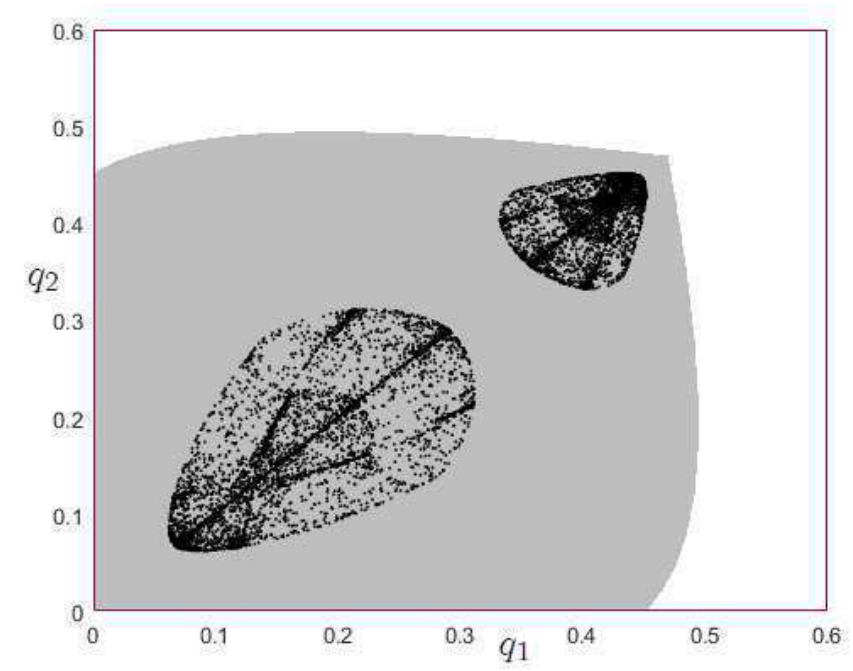

(a)

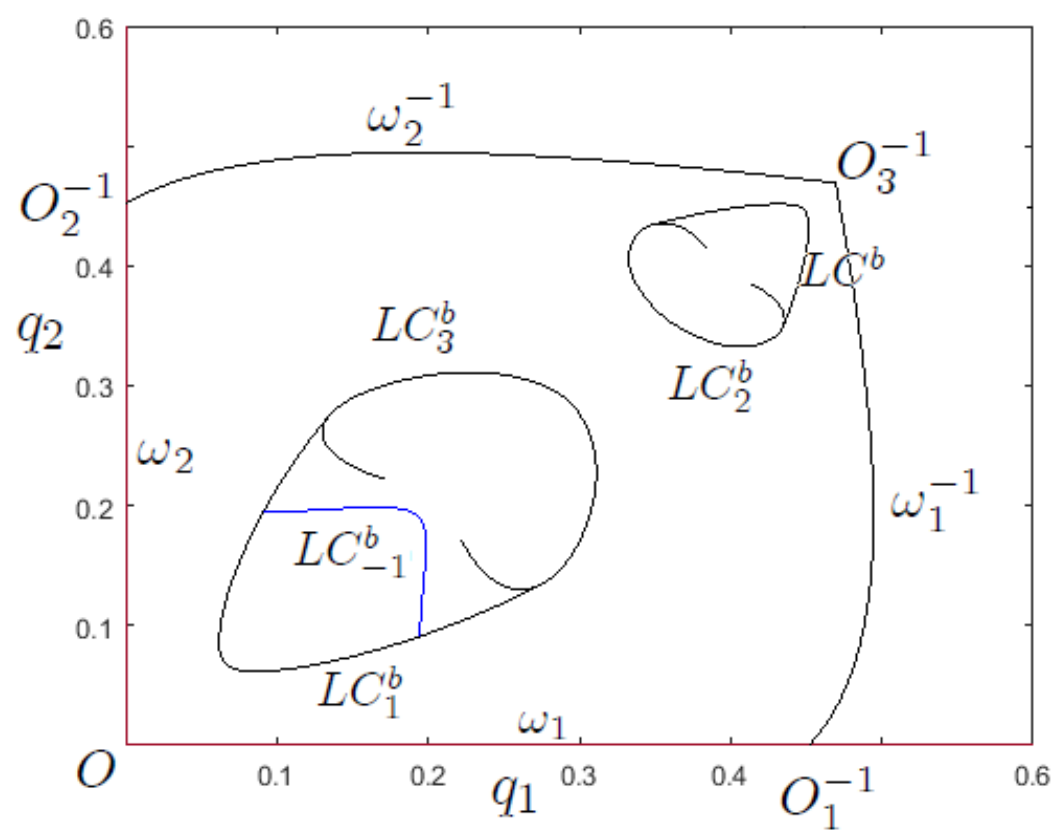

(b)

Figure 3. (a) A chaotic attractor for $\alpha=2.48, w=0.5, b=0.375$ and $d=0.35$. (b) Boundary of the attractor of map $T$, which is obtained for the same parameter values by iterating three times a segment of curve $L C_{-1}$. The basin of attraction is bounded by segments $\omega_{i}=\left[O, O_{i}^{-1}\right]$ and their rank-1 preimages. The intersection between $\omega_{1}^{-1}$ and $\omega_{2}^{-1}$ is $O_{3}^{-1}$, which is a preimage of $O$.

Such a change in long-term outcomes can be better understood by the study of the transverse Lyapunov exponent and the corresponding bifurcation diagram. Figure 4(a) shows the bifurcation 
diagram allowing us to detect the existence of a chaotic attractor on the diagonal for $d \in[0.2,0.4]$, except for some windows of low-period cycles (corresponding to which the Lyapunov exponent plotted in the figure can be interpreted neither as the natural one nor a good approximation of it). It needs mentioning that in the parameter interval we considered, the sign of the Lyapunov exponent changes several times (Figure 4(b)). This implies that small changes in $d$ can generate sharp changes in long-term dynamics. Therefore, economies starting with a similar initial condition may end up with a different long-term outcome.

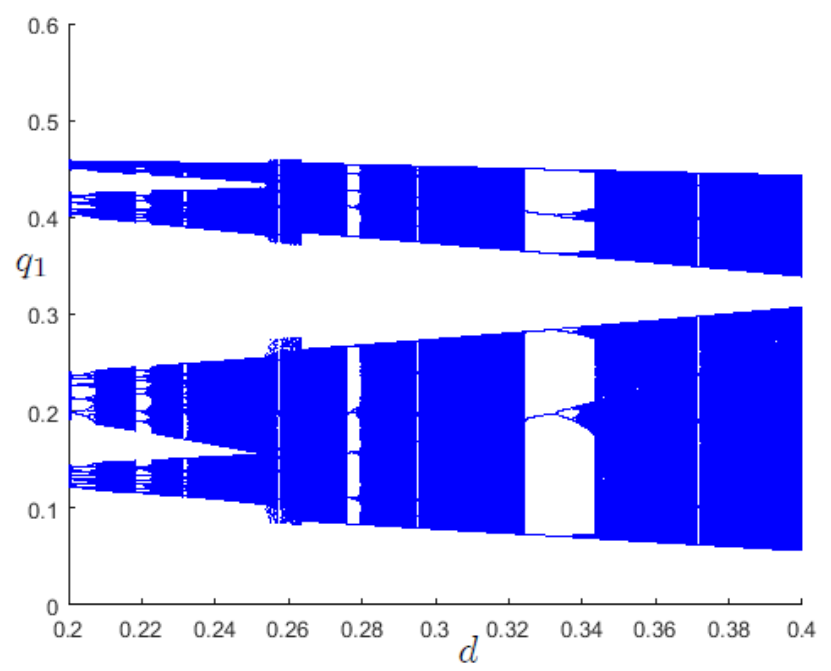

(a)

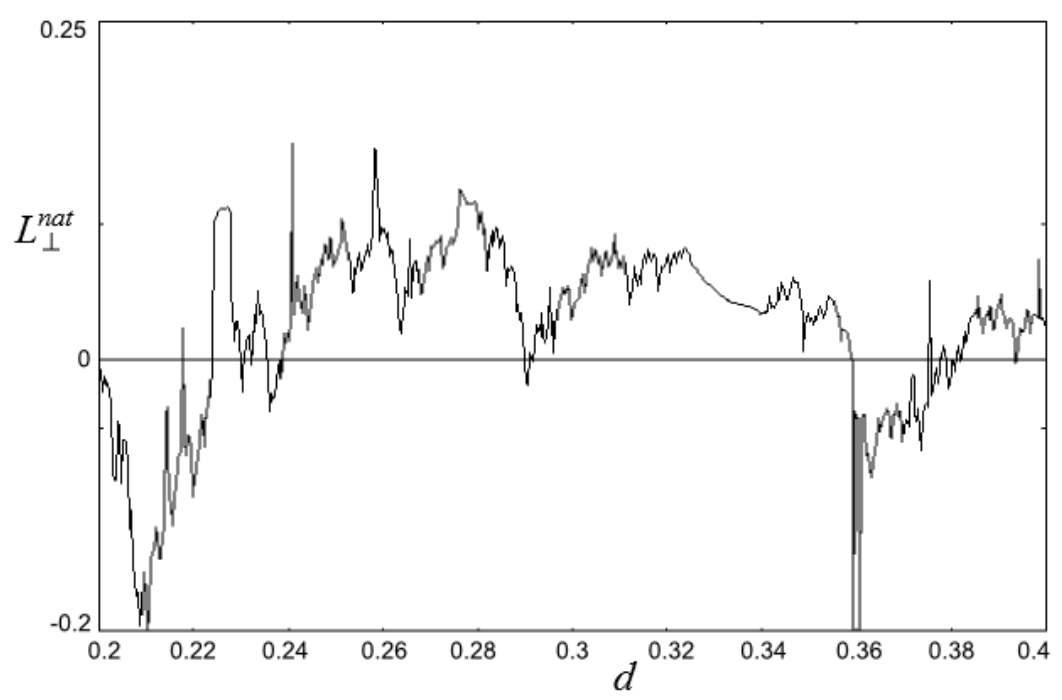

(b) 
Figure 4. (a) Bifurcation diagram for $d$. (b) Transverse Lyapunov exponent for $d \in[0.2,0.4]$. Other parameter values are: $\alpha=2.48, w=0.5, b=0.375$.

If we further increase the degree of product differentiation (i.e. we decrease the value of $d$ ), we observe coexistence of a two-period cycle along with a four-piece chaotic attractor (Figure 5(a)) outside the diagonal, which is symmetric with respect to it. Then, by letting $d$ decreasing further, it is possible to note the existence of a four-piece attractor along the diagonal, a two-period cycle of and a four-piece chaotic attractor (Figure 5(b)). Also in this case, the attractor along the diagonal is only in the Milnor sense.

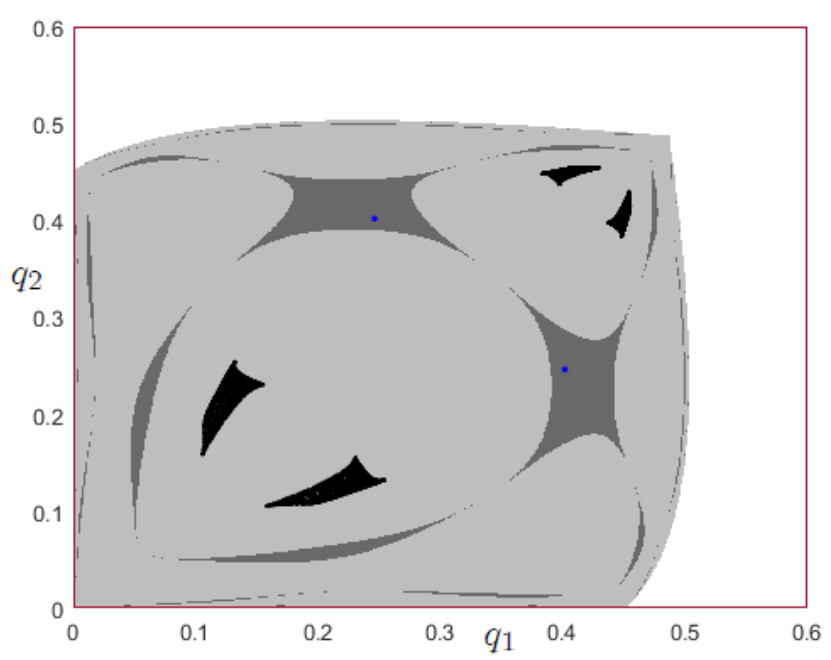

(a)

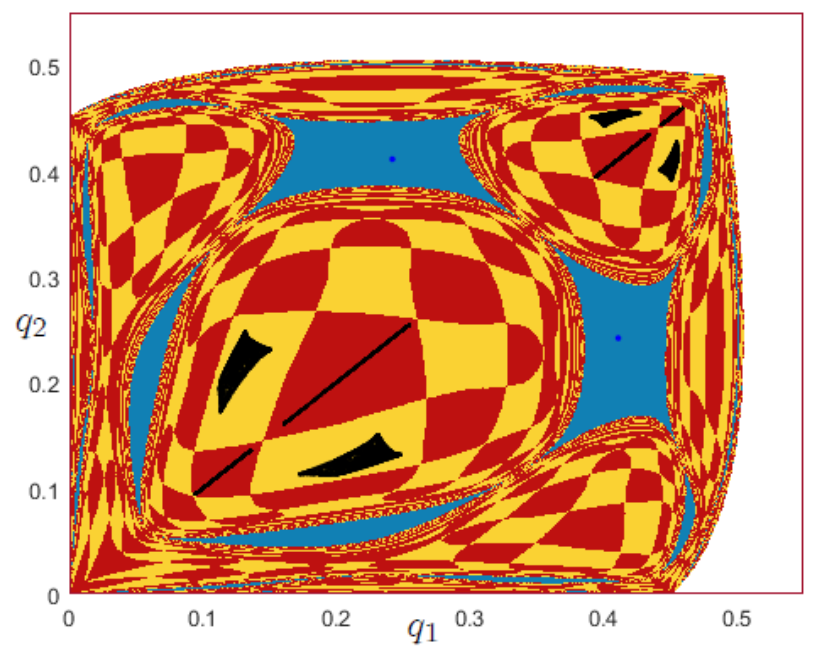

(b) 
Figure 5. (a) Coexistence of a two-period cycle and a four-piece chaotic attractor for $d=0.25$. (b) A four-piece Milnor attractor exists along the diagonal (whose basin of attraction is blood-redcoloured) together with a stable two-period cycle (whose basin of attraction is blue-coloured) and a four-piece chaotic attractor symmetric with respect to the diagonal (whose basin of attraction is yellow-coloured) for $d=0.23$. Other parameter values are: $\alpha=2.48, w=0.5$ and $b=0.375$.

\subsection{The role of the weight of managerial power in the dynamics}

Interesting phenomena can be observed if we let parameter $b$ vary by fixing $\alpha=2.48, w=0.5$ and $d=0.25$. We begin this section by considering the case in which the market share bonus is relatively small $(b=0.1)$ and the Nash equilibrium is the unique finite distance interior attractor of the system in the positive orthant (as illustrated in Figure 6(a)). An increase in $b(b=0.25)$ implies that $E^{*}$ loses stability through a period doubling bifurcation that gives rise to a stable two-period cycle along the diagonal (Figure 6(b)). A further dynamic scenario occurs if we let $b$ increase further $(b=0.35)$. To this purpose, Figure 6(c) displays initial conditions that converge to a four-period cycle on the diagonal (light gray color) and initial conditions converging to a four-period cycle out of the diagonal (dark gray color). It is interesting to note that, due to the strong nonlinearities of the model, by letting $b$ vary we observe several transverse stability switchings of the cycles embedded in the diagonal. Observe that an economy starting far away from the diagonal may synchronise, as the basin of attraction of the attractor on the diagonal is composed by several non-connected sets. An additional increase in $b$ implies that the cycle out of the diagonal undergoes a Neimark-Sacker bifurcation and a four-piece quasi-periodic attractor is born coexisting together with an attractor along the diagonal. Figure 6(d) shows the phase plane for a value of $b(b=0.367)$ far enough away from the Neimark-Sacker bifurcation one. We can see that the curves forming the four-pieces attractor have lost their properties of differentiability (see Mira et al., 1996 for details) and also that there exists an eight-piece chaotic attractor along the diagonal (created via the well-known sequence of period doubling bifurcations) and a two-period cycle. 


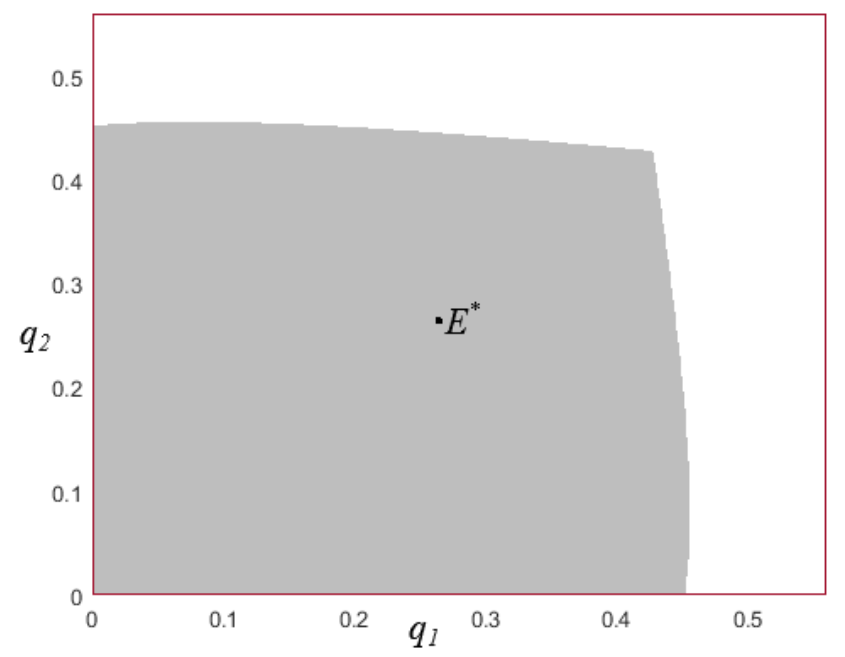

(a)

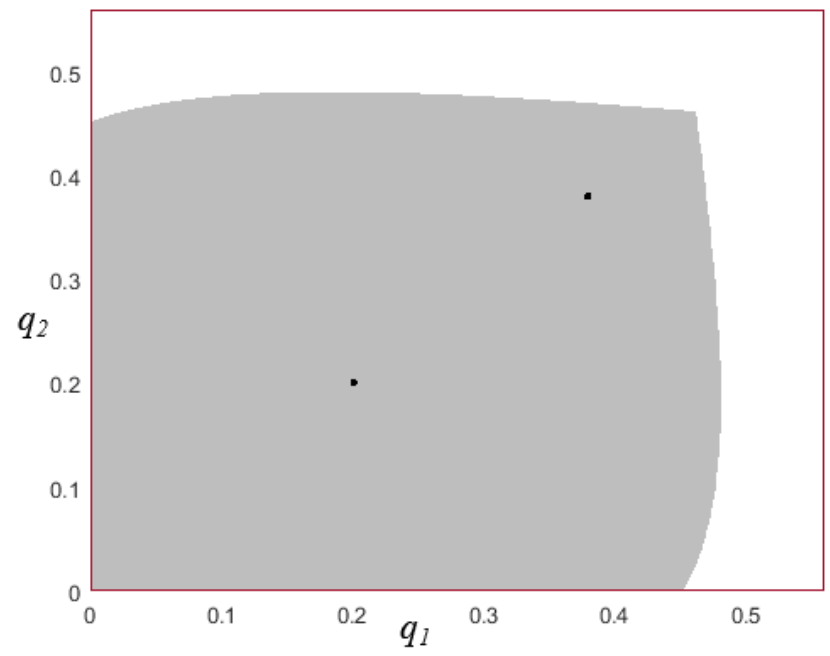

(b) 


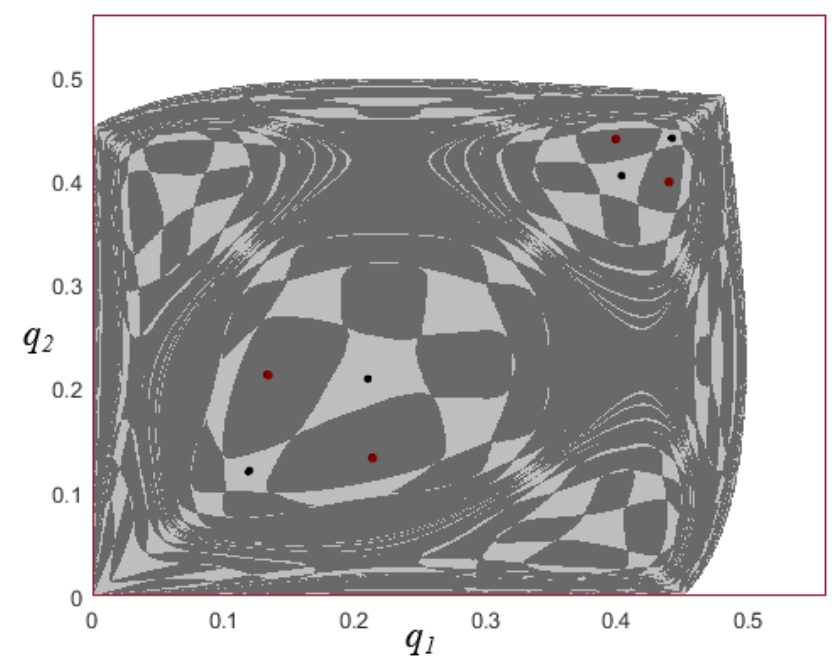

(c)

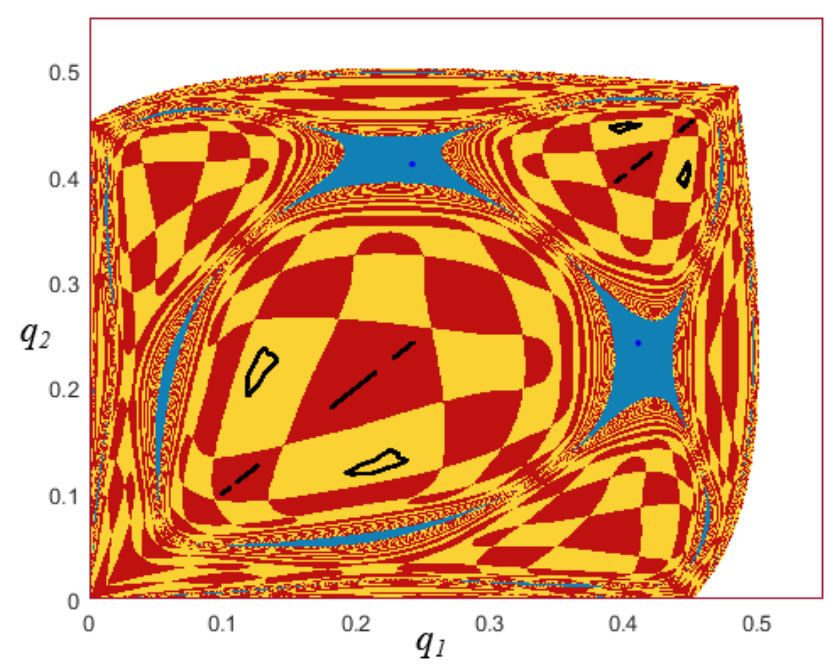

(d)

Figure 6. (a) Basin of attraction of $E^{*}$ for $b=0.1$. (b) A stable two-period cycle for $b=0.25$. (c) Two coexisting four-period cycles and their basins (depicted in light and dark gray) for $b=0.35$. The black (resp. red) two-period cycle is the attractor along (resp. out of) the diagonal and its basin of attraction is light (resp. dark) gray coloured. (d) For $b=0.367$, four attracting closed invariant curves have been created out of the diagonal (yellow basin of attraction) and coexist with an eightpiece chaotic attractor along the diagonal (blood-red basin of attraction) and a stable two-period cycle (blue basin of attraction). Other parameter values are: $\alpha=2.48, d=0.25$ and $w=0.5$.

Increasing the weight $b$ has the effect of turning the four invariant curves into a four-pieces chaotic attractor out of the diagonal. A subsequent contact bifurcation between the attractor along 
the diagonal and its basin boundary leaves only the four-piece chaotic attractor to coexist with the two-period cycle. The last two evolutions in the dynamics of the model have not been reported in any figure as they are similar to those reported in Figure 5(b) and Figure 5(a), respectively. A further increase in the value of $b(b=0.4)$ produces a union in the chaotic attractor along the diagonal and the four-piece one out of the diagonal, so that a unique two-pieces chaotic attractor eventually coexists with a two-period cycle out of the diagonal. This phenomenon is reported in Figure 7(a). We note that the attractor is close to the boundary of the basin of attraction of the two-period cycle. A further increase of $b$ creates a collision between the two sets and the chaotic attractor becomes a chaotic repellor. In this case, the unique survived attractor is the two-period cycle. It is interesting to note that in spite of the simplicity of the phase plane shown in Figure 7(b), the dynamics starting from initial conditions belonging to the area close to the chaotic repellor show a very long and erratic transient (Figure 7(c)). This fact has noteworthy economic implications related to the length of the convergence towards the two-period cycle.

Another phenomenon, which is closely related to the non-invertibility of the map, is shown in Figure 8 (a) (plotted for $b=0.45$ ). Specifically, the increase in $b$ causes a north-east shift of one of the two branches of the $L C$ curve. The contact (first) and the subsequent crossing of the boundary of the basin of attraction of the two-period cycle causes the birth of infinite portions of the basin of attraction of infinity within the region with vertices $O, O_{1}^{-1}, O_{3}^{-1}$ and $O_{2}^{-1}$ (see also the enlargement view reported in Figure 8(b)).

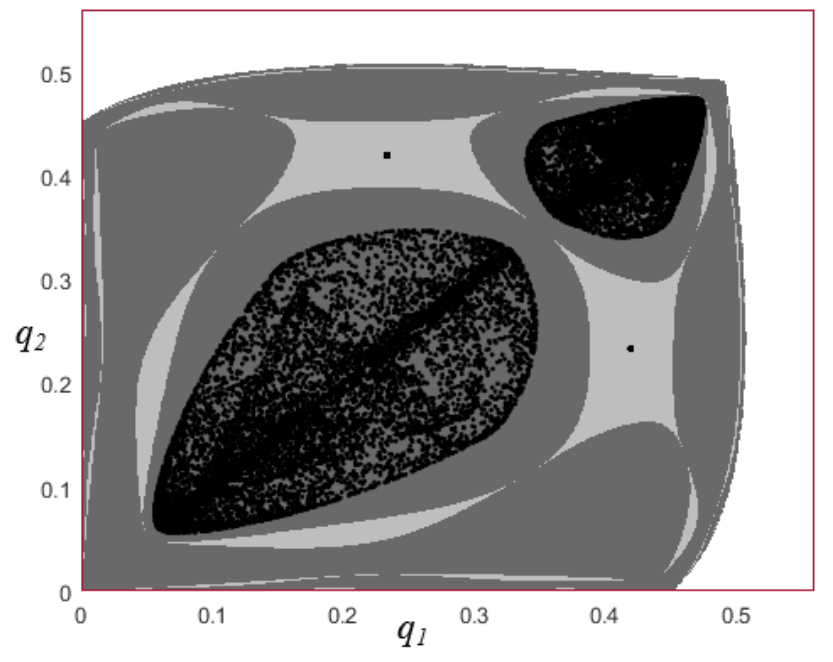

(a)

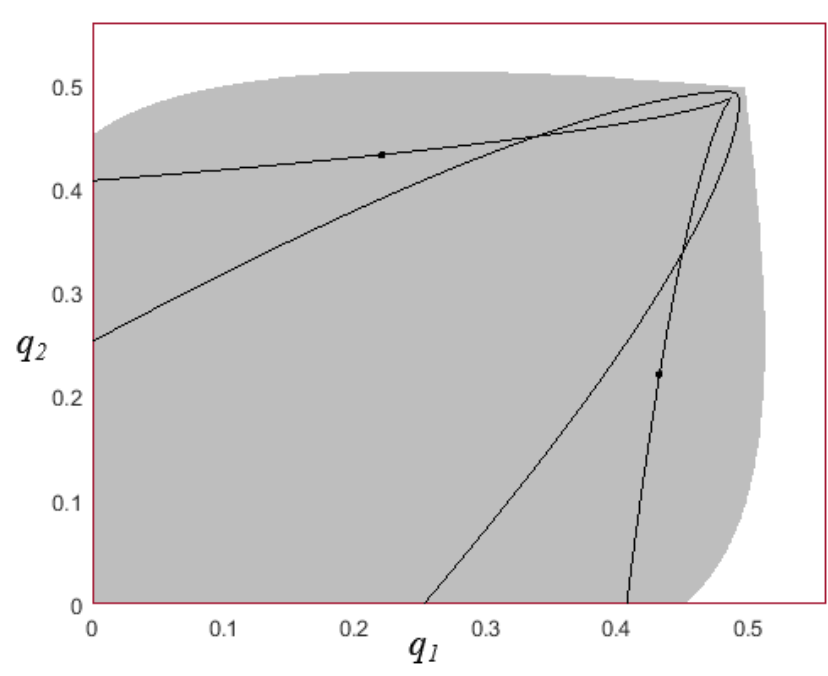

(b) 


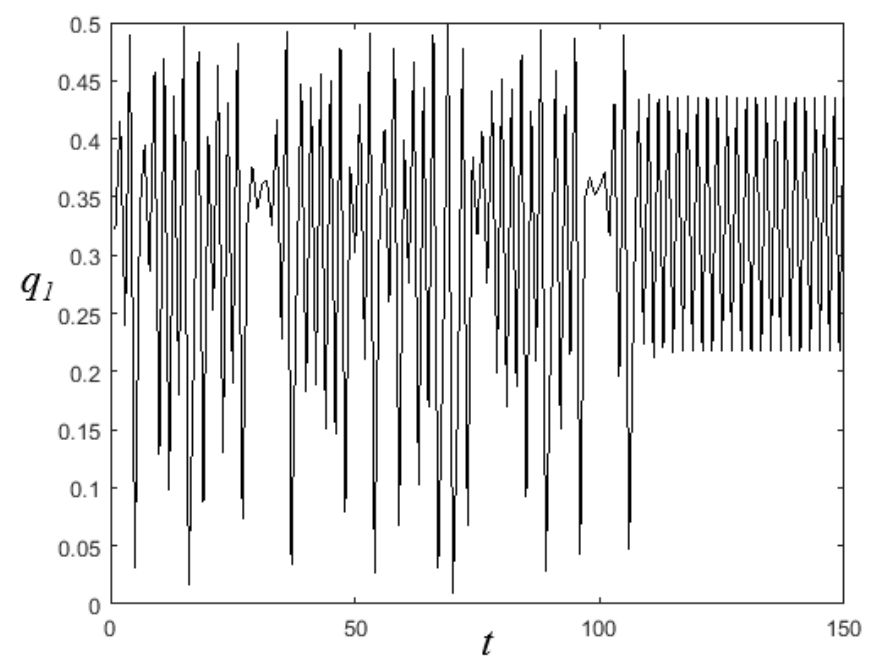

(c)

Figure 7. (a) For $b=0.4$ a two-piece chaotic attractor along the diagonal coexists with a stable two-period cycle out of the diagonal. (b) A further increase of $b$ turns out the chaotic attractor into a chaotic repellor and the unique survived attractor is the two-period cycle. (c) The dynamics starting from initial conditions belonging to the area close to the chaotic repellor show a very long and erratic transient. Other parameter values are: $\alpha=2.48, d=0.25$ and $w=0.5$.

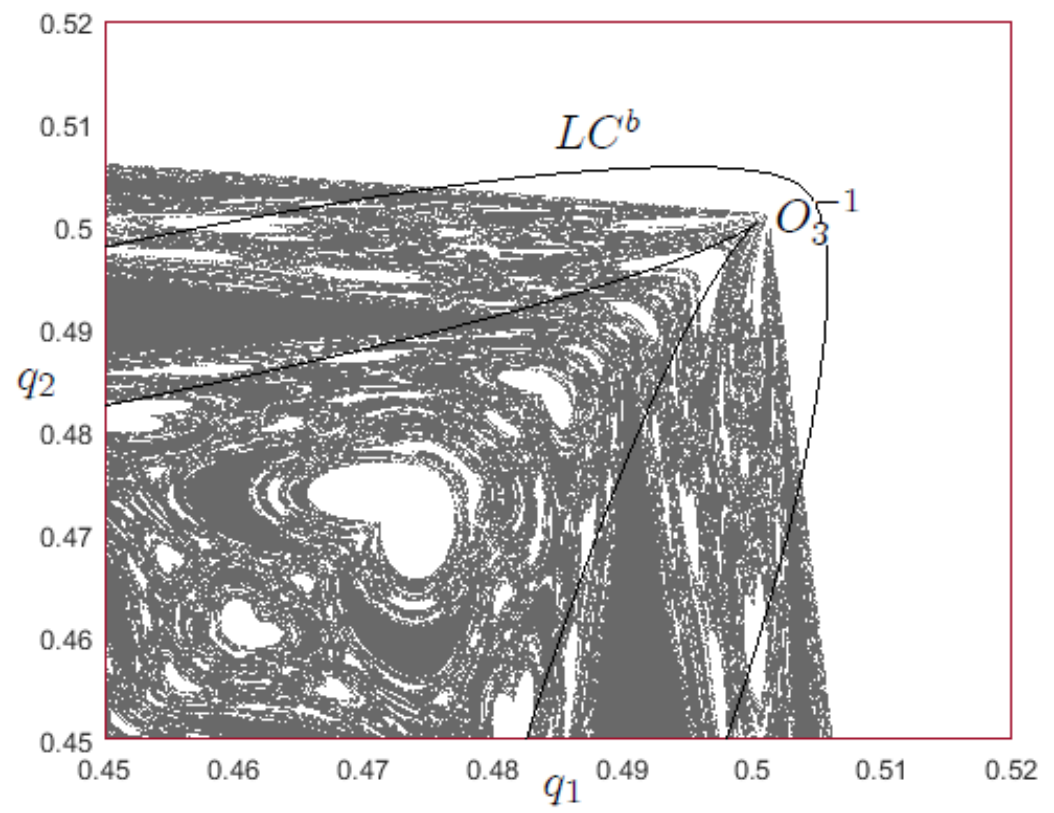

(a) 


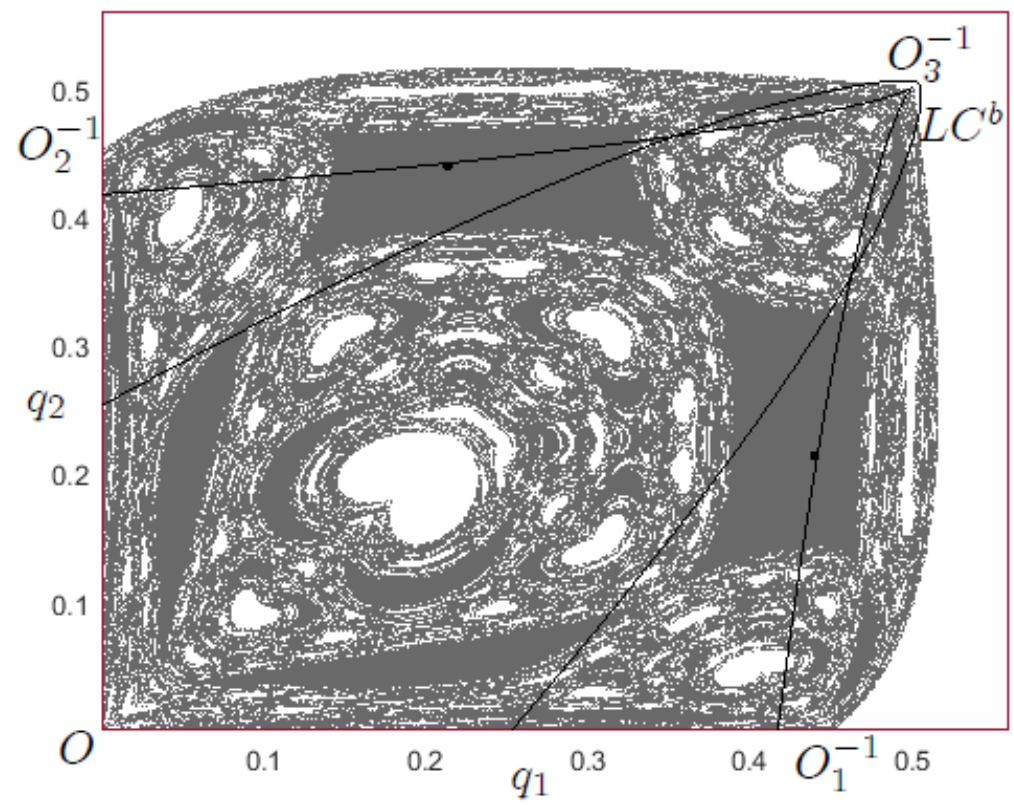

(b)

Figure 8. (a) Infinitely many holes appear in the basin of attraction of the two-period cycle for $b=0.45$. $O_{1}^{-1}, O_{3}^{-1}$ and $O_{2}^{-1}$ are the pre-images of $O$. (b) Enlargement view: the contact and the subsequent crossing of the boundary of the basin of attraction of the two-period cycle causes the birth of infinite portions of the basin of attraction of infinity. Other parameter values are: $\alpha=2.48$, $d=0.25$ and $w=0.5$.

\section{$5 \quad$ Entrant and incumbent}

In the previous sections, we have studied the dynamic evolution of trajectories that lie in the positive orthant for every iterate. We recall that from a dynamic point of view, an initial condition lying in the white region of the basins of attraction (depicted in the figures previously shown), generates a trajectory for which there exists at least an iterate such that the quantity produced by one of the two firm is negative. We note that in the cases illustrated in all the figures except the cases of Figures $9(\mathrm{a})$ and 9 (b), the white region falls within the $Z_{0}$ area of the map. Then, trajectories starting from initial conditions that belong to the white region exit the positive orthant in one iterate. Instead, the situation shown in the example of Figure 8(a) is different. This because the existence of infinitely many holes in $Z_{2}$ area can generate trajectories that take more than one iterate to enter the negative region.

In the seminal article of Bischi et al. (1998), trajectories leading towards the negative region (of the quantity produced at least by one of the two firms) have been interpreted as having no economic sense and then removed from the analysis. However, another approach proposed in the literature is the one of Fanti et al. (2012), where it has been considered an adjustment mechanism of quantities 
such that

$$
q_{i}^{\prime}=\max \left\{0, q_{i}+\alpha q_{i} \frac{\partial \Pi_{i}}{\partial q_{i}}\right\}, \quad i=1,2,
$$

where $\partial \Pi_{i} / \partial q_{i}$ represents the marginal profit of firm $i$. The implications of the specification summarised in (16) have been deepened in the work of Fanti et al. (2015). Specifically, the economic interpretation given by the authors to such a model is that a trajectory taking negative values implies that at least one of the two firm exits the market. If only one of them actually exits, the rival becomes a monopolist and - depending on parameter specifications - its production will tend to be the equilibrium value of a monopolist output or, alternatively, it may fluctuate over time (due to the assumption of limited information on the market demand) if the reactivity of the firm is large enough. Although this result is coherent with the dynamic system proposed, there may be some problems related to its economic interpretation. In fact, the fixed point of a dynamic system that lies on one of the axes may not be a Nash equilibrium. Then, it may be profitable for the firm that previously decided to exit to re-enter the market.

This kind of problem can actually be related to that of choosing whether it is convenient for one player (the entrant) to enter a market where there is already another player (the incumbent). This topic has formerly been studied, for instance, by Cánovas et al. (2008) in a context where players (firms) have perfect knowledge of the market demand and decision rules are based on the best reply (Puu, 1991). However, the model developed in the article exhibits marked differences compared to Cánovas et al. (2008). First, there exists a market for (potentially two distinct) heterogeneous products (as they are perceived by consumers), whereas Cánovas et al. (2008) considers a market for a single homogeneous good. Then, there actually exist two different market curves, each of which directed to one specific firm. This implies the existence of two different (non-separated) markets. Second, we have considered players with limited information. Specifically, firms do not know the market demand and - as they cannot compute their own best reply - use a behavioural rule to produce next period output (Bischi et al., 1998).

Let us now modify map $T$ in order to account for the possibility that a firm that temporarily closed down (that is, it has played the quantity $q_{i}(t)=0$ at time $t$ ) wants to re-enter the market by producing a strictly positive quantity after a time of inaction of $k \geq 0$ periods. In particular, let us assume that the entrant enters with a kind of "initial condition" $\varepsilon>0$, whose extent is fixed by a rule of thumb. Mathematically, let us introduce the following map:

$$
T^{*}=\left\{\begin{array}{c}
q_{1}(t+1)=\bar{T}_{1}^{*}\left(q_{1}(t), q_{2}(t), l_{1}(t)\right) \\
l_{1}(t+1)=L_{1}\left(q_{1}(t), l_{1}(t)\right) \\
q_{2}(t+1)=\bar{T}_{2}^{*}\left(q_{2}(t), q_{1}(t), l_{2}(t)\right) \\
l_{2}(t+1)=L_{2}\left(q_{2}(t), l_{2}(t)\right)
\end{array}\right.
$$

where

$$
\begin{aligned}
& \bar{T}_{i}^{*}\left(q_{i}(t), q_{-i}(t), l_{i}(t)\right)=\left\{\begin{array}{c}
0 \text { if } 0<l_{i}(t)<k \\
\varepsilon \text { if } l_{i}(t)=k \\
r_{i}\left(q_{i}(t), q_{-i}(t)\right) \text { if } l_{i}(t)=0
\end{array},\right. \\
& L_{i}\left(q_{i}(t), l_{i}(t)\right)=\left\{\begin{array}{ccl}
0 & \text { if } \quad q_{i}(t)>0 & \text { or } \quad l_{i}(t)=k \\
& l_{i}(t)+1 \quad \text { if } & q_{i}(t)=0
\end{array},\right.
\end{aligned}
$$


and

$$
r_{i}\left(q_{i}(t), q_{-i}(t)\right)=\max \left\{0, q_{i}(t)+\alpha q_{i}(t)\left[1-2 q_{i}(t)-d q_{-i}(t)-w+b \frac{q_{-i}(t)}{\left(q_{i}(t)+q_{-i}(t)\right)^{2}}\right]\right\},
$$

$i=1,2$. Equation $l_{i}(t+1)=L_{i}\left(q_{i}(t), l_{i}(t)\right)$ counts the consecutive periods of inactivity of firm $i$ and it is zero if the quantity produced is positive. Based on the values of this equation it is defined the expression in (18), the value of which gives us the quantity produced at the subsequent period.

In the example illustrated in Figure 6(a), the adjustment mechanism just described by map $T^{*}$ in (17) actually enlarges the basin of attraction of the Nash equilibrium by including all regions of the positive orthant below the demand curves (as is shown in Figure 9(a) for any value of $k$ ). Differently, by considering the parametric specification of Figure 6(d), implying coexistence of attractors for map $T$, it is more difficult to understand how the re-injection mechanism (the quantity produced by the entrant, i.e. $\varepsilon$ ) modifies long-term trajectories of initial conditions belonging to the white region of Figure 6(d). Figure 9(b) shows, for $\varepsilon=0.2$ and $k=1$, that all the initial conditions that generated an unfeasible trajectory under map $T$ now generate trajectories convergent to the eight-piece chaotic attractor under map $T^{*}$. From a visual point of view, the white region in Figure 6(d) has become redcoloured in Figure 9(b). Therefore, the ex white region has become part of the basin of attraction of the eight-piece chaotic attractor. More difficult is instead the analysis of this specific kind of reinjection rule associated with the case depicted in Figure 8(a). In fact, the existence of infinitely many holes in $O O_{1}^{-1} O_{3}^{-1} O_{2}^{-1}$ area (portions of basin of attraction of infinity) actually causes the appearance of temporary entry barriers. Therefore, it is possible to observe that some attempts of the entrant to re-enter the market can generate a long-term result such that 1) both firms produce strictly positive quantities (in this case trajectories converge towards the two-period cycle), or 2) at least one firm alternates periods in which its production is strictly positive to periods in which it closes down. To this purpose, we propose an explicit method to prove the existence of a set of trajectories such that one of the two firms chooses to temporarily exit the market, that is there are several periods in which the market is characterised by a monopoly (as is shown in Figure 9(c)). We note that the restriction of map $T$ on one of the two axes is conjugate to the logistic map. For the parameter set used to plot Figure 8(a), the stationary equilibrium of such a map attracts all points starting from the same axis. Therefore, if the length of time of inactivity of the closing firm is sufficiently long, the production level of the incumbent will be close enough to the stationary value $\frac{1-w}{2}$. Now, by considering a value of $\varepsilon$ for which an iterate belongs to one of the holes existing in Figure 8(a), we will have a trajectory characterised by some iterates for which the quantity produced by one of the two firms is zero and the quantity produced by the other firm is positive, and some iterates for which the quantities produced by both firms are positive. By generalising these arguments, it is possible to build on the whole basin of attraction of this kind of trajectories, which is reported in Figure $9(\mathrm{~d})$. In particular, the figure shows that the trajectories starting from the blue region imply that for some periods one of the two firms exit the market whereas the other one becomes a monopolist. Differently, the yellow region describes the case in which firms enter and exit together, whereas the red region represents initial conditions for which both firms produce in the long term by converging towards the two-period cycle.

Another way of modelling the re-entering mechanism by one of the two firm is to assume (given the hypothesis of limited information) that a firm tries to re-enter the market randomly after a possible 
time of inaction. Therefore, if by relaxing the assumption of symmetric and constant entry and assuming that firms enter the market with a random production level, nothing changes with respect to the case of deterministic entry if we consider the parametric example of Figure 6(a). Instead, when there are multiple attractors or holes in the basin of attraction we may have rather different dynamic events in comparison with the deterministic case. In particular, by considering the parameter set of Figure 8(a) under random entry, market dynamics become intense and characterised by periods in which one of the firm is out of the market (as is shown in Figure 10). In this sense, the existence of holes in the basin of attraction is just a signal of (dynamic) entry barriers and market instability.

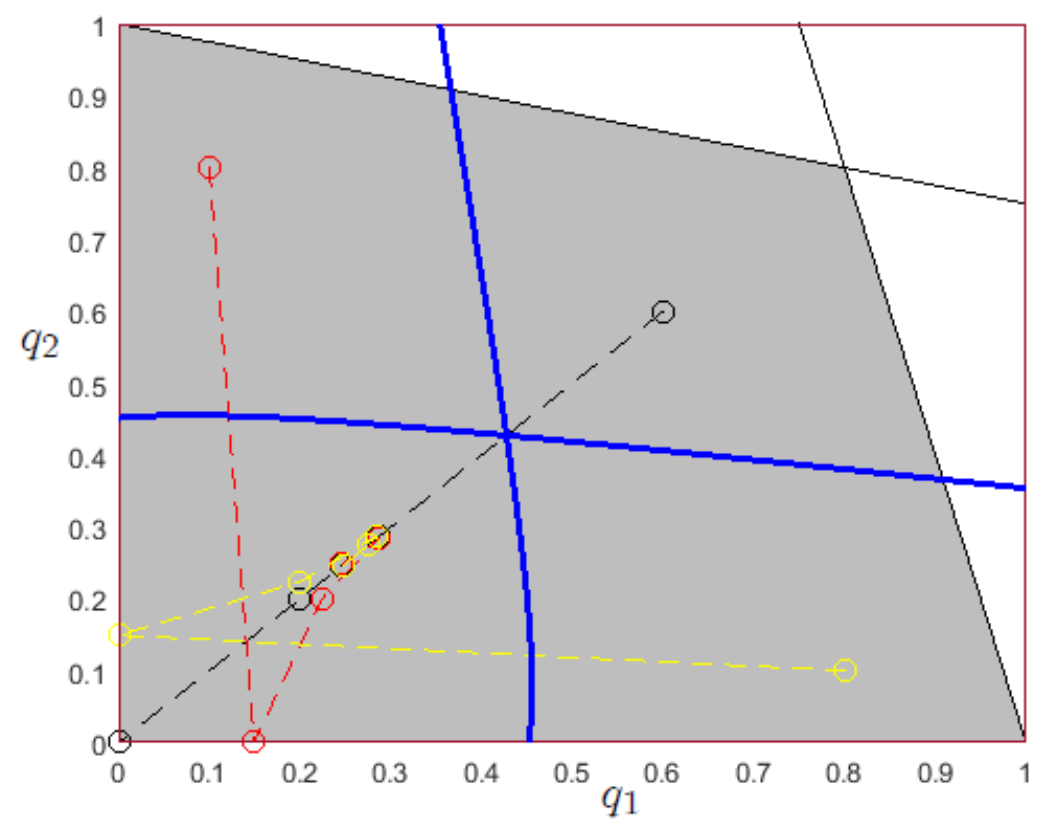

(a) 


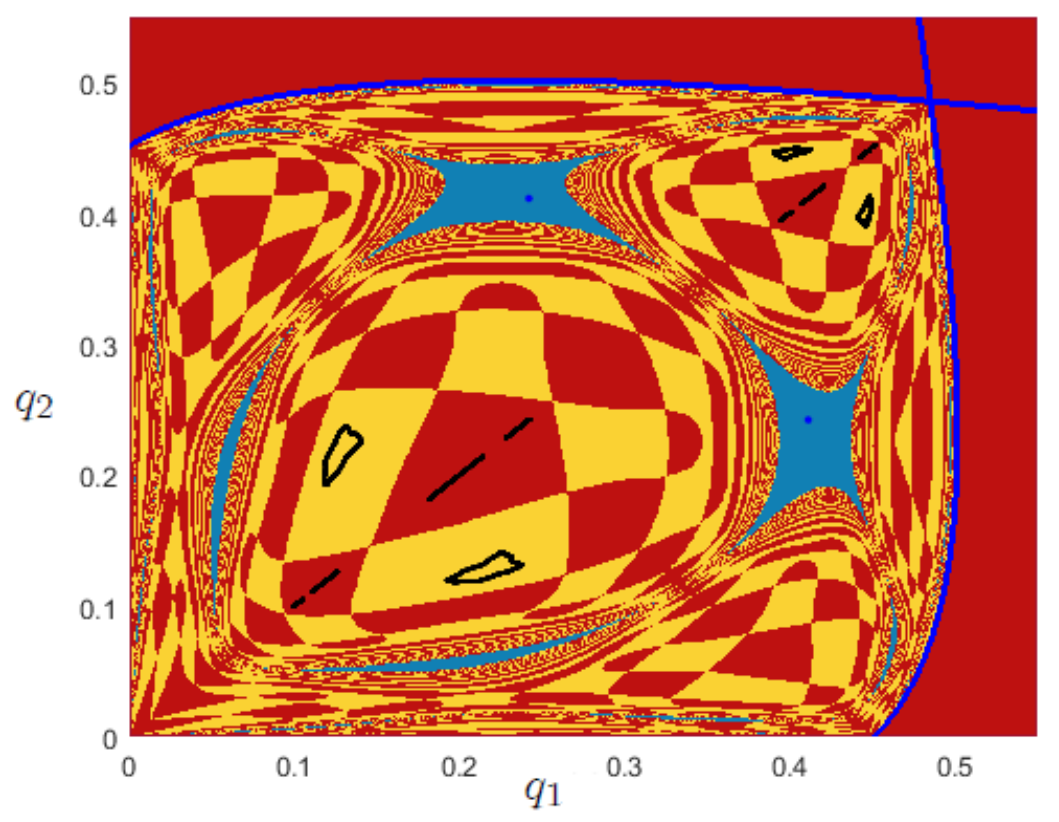

(b)

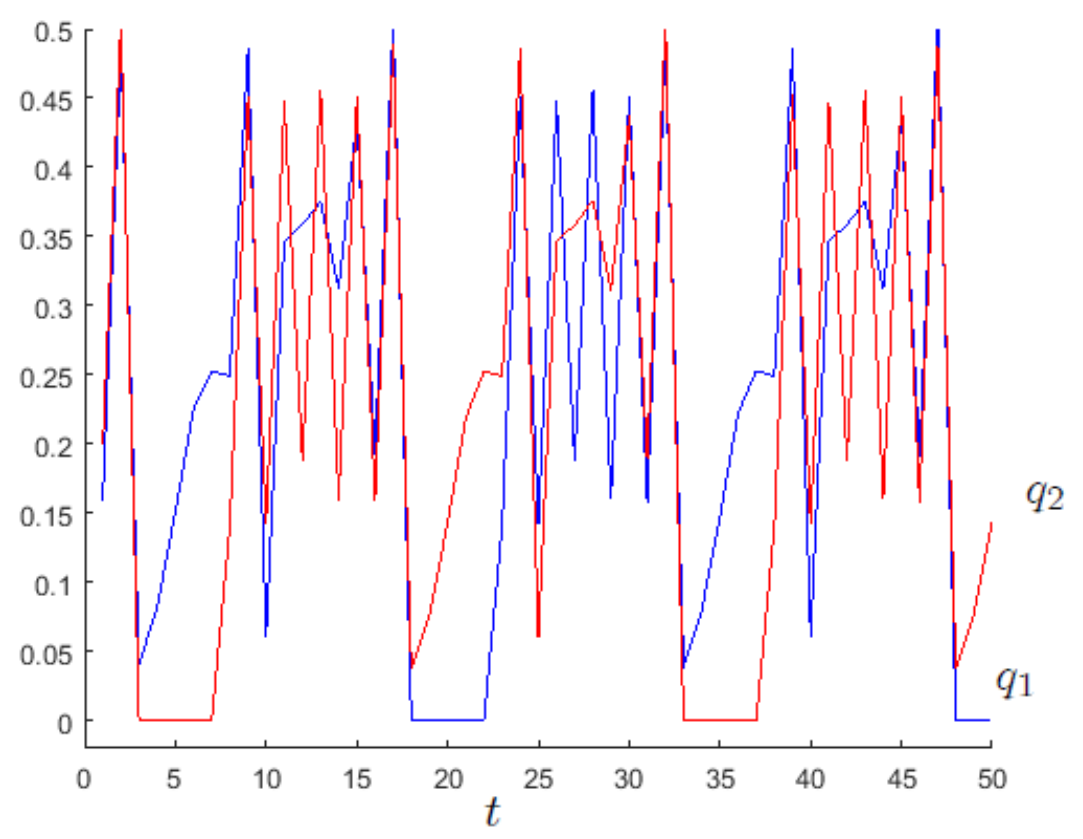

(c) 


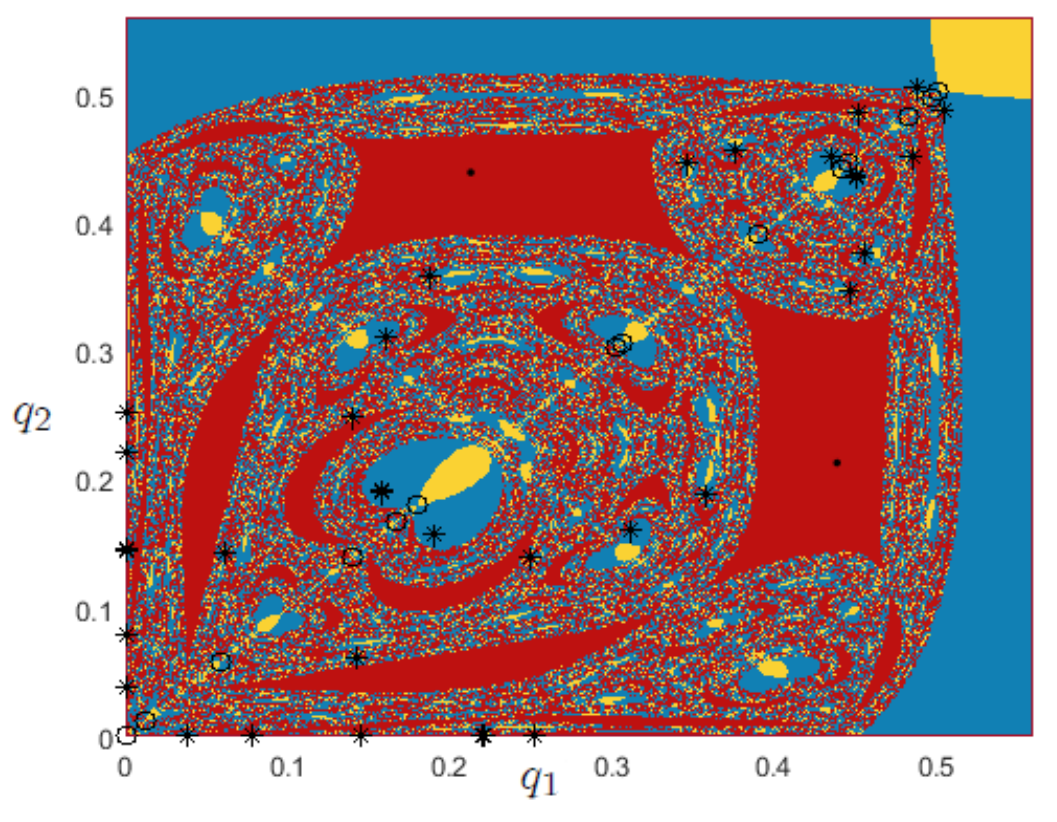

(d)

Figure 9. Basins of attraction with the reinjection mechanism described by map $T^{*}$. The blue lines in the figures separate the regions in which an iterate remains positive or it jumps directly on the axes. The black lines with negative slopes separate the regions in which prices both both products are positive (grey) to regions in which prices are negative (white). (a) The parameter set is the same as in Figure 6(a). The (red, black and yellow) dotted lines depict three different trajectories. These trajectories start by initial conditions that, from an economic point of view, would become unfeasible in a context without the re-injection mechanism. (b) The parameter set is the same as in Figure 6(d). (c) Trajectories with an entry/exit process from the market of one of the two firms. (d) Basins of attraction of $T^{*}$. The parameter set is the same as in Figure 8(a) with $\varepsilon=0.14$. The asterisks describe the attractor capturing all trajectories starting out from the blue region. The circles, instead, represent the attractor synchronised on the diagonal and characterised by having a market where firms that entry and exit together. The dots are the two-period cycle capturing all the trajectories within the red region. 


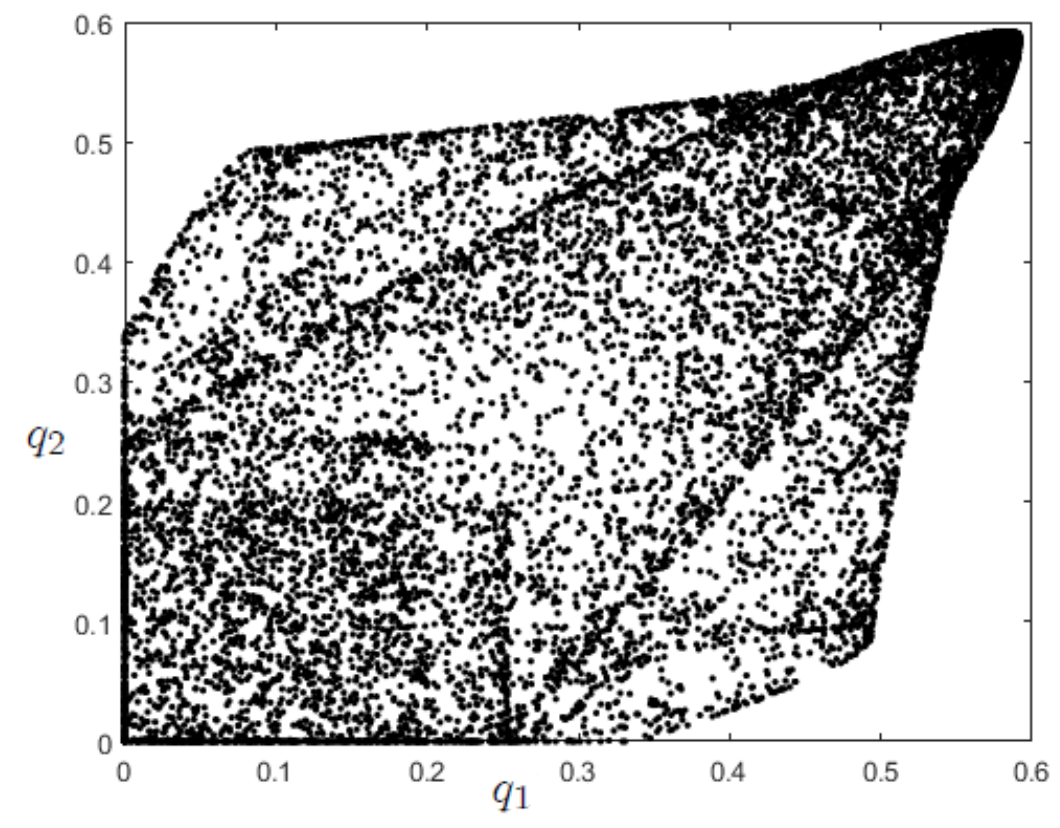

Figure 10. Typical trajectories by considering a random value of $\varepsilon$ fixed in the interval $(0,0.2)$.

In this model we have assumed that products of both firms enter in the same way (that is, they have the same weight in) the representative consumer's utility function. However, this assumption appears a bit restricting to adequately capture the behaviour of a market where there are an entrant and an incumbent. One way to overcome this limitation is to consider that the product of the entrant has a smaller weight than that of the incumbent in the utility function of the representative consumer (this may be due to habit formation in consumption, commodities characteristics, etc.). In addition, if the entrant is able to stay in the market for a relatively long time period, such a weight will tend to be equal to the one related to the product of the incumbent. From a mathematical point of view, by assuming that the entrant (firm 2 ) enters the market at time $t_{0} \geq 1$, we can generalise the representative consumer's optimisation problem by starting from the definition of a utility function that accounts for differences in product characteristics as perceived by the consumer. Therefore, utility function $U\left(q_{1}, q_{2}\right)$ will now be specified as follows:

$$
U\left(q_{1}, q_{2}\right)=q_{1}+q_{2}-\frac{1}{2}\left(\beta_{1} q_{1}^{2}+\beta_{2} q_{2}^{2}+2 d q_{1} q_{2}\right)
$$

where $\beta_{1}$ and $\beta_{2}$ represent two distinct parameters that are negatively correlated to the weights attached to products 1 and 2, respectively, by the consumer. By solving the maximisation problem of the agent, we get the indirect demand function of product $i$, that is $p_{i}=1-\beta_{i} q_{i}-d q_{-i}(i=1,2)$. We now assume that $\beta_{1}:=1$ and $\beta_{2}:=1+\frac{y}{t-t_{0}+1}(y \geq 0)$, implying that the demand of product of firm 1 has a constant steepness (normalised to 1 ) and the demand of product of firm 2 has a time-varying steepness. We note that with such a parametric specification, the values of $\beta_{1}$ and $\beta_{2}$ 
will tend to be equal $\left(\beta_{2} \rightarrow \beta_{1}\right)$ if both firms stay together in the market for a sufficiently long time period, that is the consumer will tend to weight the consumption of product 1 and the consumption of product 2 in the same way when time goes by.

By considering a dynamic setting and avoiding replicating all the mathematical steps previously done, the two-dimensional dynamic system is now described by the following non-autonomous map:

$$
T^{* *}:\left\{\begin{array}{c}
q_{1}(t+1)=q_{1}(t)+\alpha q_{1}(t)\left[1-2 q_{1}(t)-d q_{2}(t)-w+b \frac{q_{2}(t)}{\left(q_{1}(t)+q_{2}(t)\right)^{2}}\right], \\
q_{2}(t+1)=S\left(q_{1}(t), q_{2}(t), t\right)
\end{array}\right.
$$

where

$S\left(q_{1}(t), q_{2}(t), t\right)=\left\{\begin{array}{c}0 \quad \text { if } t<t_{0} \\ \varepsilon \quad \text { if } t=t_{0} \\ q_{2}(t)+\alpha q_{2}(t)\left[1-2\left(1+\frac{y}{t-t_{0}+1}\right) q_{2}(t)-d q_{1}(t)-w+b \frac{q_{1}(t)}{\left(q_{1}(t)+q_{2}(t)\right)^{2}}\right] \quad \text { if } \quad t>t_{0}\end{array}\right.$.

Our aim in this section is not to develop and disentangle all the details of map $T^{* *}$ as it does go beyond the scope of the present article (see Panchuk and Puu, 2009, for an analysis of a time-varying oligopoly model). However, by considering the numerical specification $\alpha=0.48, b=0.1, w=0.5$ and $d=0.25$, we note that the existence of a time-dependent parameter $\left(\beta_{2}\right)$ actually generates a further source of entry barriers for the potential entrant. Let us now assume that at time $t_{0}=0$ there exists only one incumbent (firm 1) in the market (monopoly). By looking at such a situation, a potential entrant (firm 2) tries to produce a rival good and then enters the market at time $t_{0}=1$. The following figures show that high values of $y$ can actually generate entry barriers (that is, due, for instance, to consumption habits so that the weight attached to the product of the entrant in the utility function of the consumer is sufficiently low). In fact, for $y=1.1$ both firms produce in the long term (Figure 11(a)), whereas when $y=12.1$ firm 2 closes down (Figure 11(b)). 


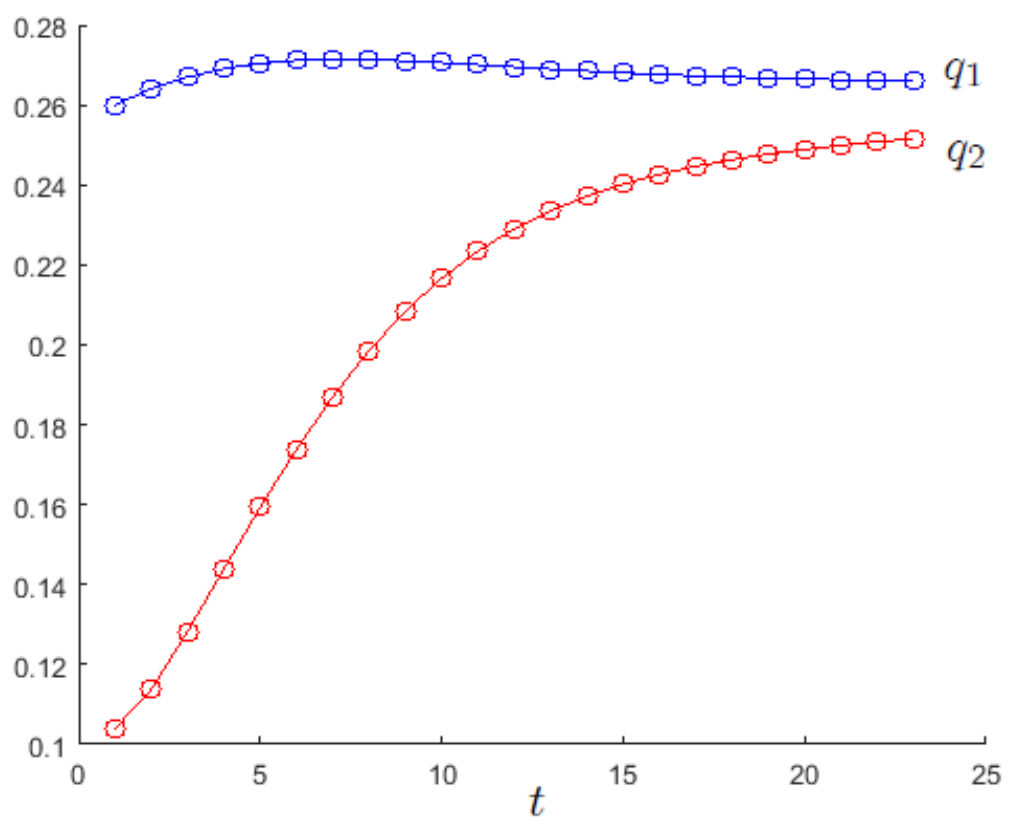

(a)

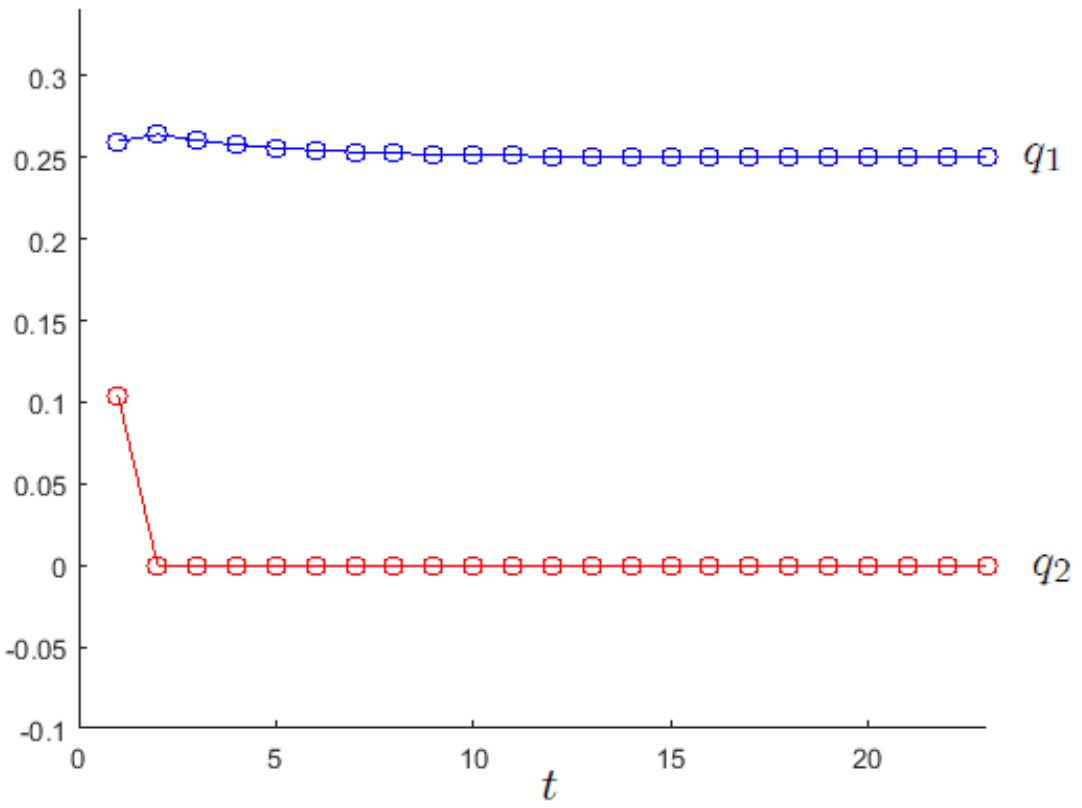

(b)

Figure 11. (a) No entry barriers $(y=1.1)$. (b) Entry barriers $(y=12.1)$. 


\section{Conclusions}

This article has analysed a dynamic repeated duopoly with quantity competition by following the tradition of Bischi et al. (1998), in which firms have limited information. Specifically, local and global dynamics have been studied by assuming managerial firms with market share delegation contracts (Jansen et al., 2007; Ritz, 2008) are horizontal product differentiation (Singh and Vives, 1984). The relationship between product differentiation in oligopoly contexts where firms are not profit maximising is the subject of a growing body of studies with the aim of ranking equilibrium outcomes in static games (Jansen et al., 2009). The importance of product differentiation as a device capturing the effects of advertising investments in the market may represent a strong empirical motivation to address this issue also in theoretical nonlinear duopolies (Gori et al., 2015), for instance to inquire about managerial behaviour over time depending on the relative degree of substitutability between products. Although advertising investments have not been explicitly modelled, this work has tackled the issue of product differentiation by considering one of the most relevant delegation contract from an empirical perspective, i.e. the market share case. By combining mathematical analysis and numerical experiments, it has presented some local and global dynamic results, showing that - depending on the relative degree of substitutability and managerial power - the Nash equilibrium of the game may not be the long-term outcome where the market converges to. In fact, it has been found that the steadystate equilibrium of the discrete time dynamic system may be unstable or other attractors (simple or chaotic) may capture long-term economic dynamics. The article has also addressed problems related to (dynamic) entry/exit processes of a firm in the market. This topic, in fact, may be of importance in a strategic context and further analyses in this direction are of potential interest, especially with regard to models with price competition, as in Fanti et al. (2013), where product differentiation is an important characteristic from an empirical point of view (Gasmi et al., 1992).

Conflict of Interest: The authors declare that they have no conflict of interest.

Acknowledgements The authors gratefully acknowledge two anonymous reviewers for valuable comments and suggestions on an earlier draft. The usual disclaimer applies.

\section{References}

[1] Agliari A, Bignami F (2010) Synchronization and on-off intermittency phenomena in a market model with complementary goods and adaptive expectations. Stud Nonlinear Dyn E 14(2):1-15.

[2] Agliari A, Chiarella C, Gardini L (2006) A re-evaluation of adaptive expectations in light of global nonlinear dynamic analysis. J Econ Behav Organ 60(4):526-552

[3] Bebchuk L, Fried J (2004) Pay without Performance. The Unfulfilled Promise of Executive Compensation. Cambridge (MA) US: Harvard University Press

[4] Bebchuk L, Grinstein Y (2005) The growth of executive pay. Oxford Rev Econ Pol 21(2):283-303

[5] Bischi GI, Gardini L (2000) Global properties of symmetric competition models with riddling and blowout phenomena. Discrete Dynamics in Nature and Society 5(2000):149-160 
[6] Bischi GI, Gardini L, Mira C (2003) Plane maps with denominator. Part II: noninvertible maps with simple focal points. Int J Bifurcat Chaos 13(8):2253-2277

[7] Bischi GI, Mammana C, Gardini L (2000) Multistability and cyclic attractors in duopoly games. Chaos Soliton Fract 11(4):543-564

[8] Bischi GI, Naimzada AK, Sbragia L (2007) Oligopoly games with Local Monopolistic Approximation. J Econ Behav Organ 62(3):371-388

[9] Bischi GI, Stefanini L, Gardini L (1998) Synchronization, intermittency and critical curves in a duopoly game. Math Comput Simulat 44(6):559-585

[10] Cánovas JS, Puu T, Ruíz M (2008) The Cournot-Theocharis problem reconsidered. Chaos Soliton Fract 37(4):1025-1039

[11] Fama EF, Jensen MC (1983) Separation of ownership and control. J Law Econ 26(2):301-325

[12] Fanti L, Gori L, Sodini M (2012) Nonlinear dynamics in a Cournot duopoly with relative profit delegation. Chaos Soliton Fract 45(12):1469-1478

[13] Fanti L, Gori L, Sodini M (2015) Nonlinear dynamics in a Cournot duopoly with isoelastic demand. Math Comput Simulat 108:129-143

[14] Fanti L, Gori L, Sodini M (2016) Managerial delegation theory revisited. Manage Decis Econ, forthcoming

[15] Fanti L, Gori L, Mammana C, Michetti E (2013) The dynamics of a Bertrand duopoly with differentiated products: synchronization, intermittency and global dynamics. Chaos Soliton Fract $52: 73-86$

[16] Fanti L, Gori L, Mammana C, Michetti E (2014) Local and global dynamics in a duopoly with price competition and market share delegation. Chaos Soliton Fract 69:253-270

[17] Fershtman C (1985) Managerial incentives as a strategic variable in duopolistic environment. Int J Ind Organ 3(2):245-253

[18] Fershtman C, Judd K (1987) Equilibrium incentives in oligopoly. Am Econ Rev 77(5):927-940

[19] Gasmi F, Laffont JJ, Vuong QH (1992) Econometric analysis of collusive behavior in a soft-drink market. J Econ Manage Strat 1(2):277-311

[20] Gori L, Sodini M, Fanti L (2015) A nonlinear Cournot duopoly with advertising. Chaos Soliton Fract 79:178-190

[21] Jansen T, van Lier A, van Witteloostuijn A (2007) A Note on strategic delegation: the market share case. Int J Ind Organ 25(3):531-539

[22] Jansen T, van Lier A, van Witteloostuijn A (2009) On the impact of managerial bonus systems on firm profit and market competition: the cases of pure profit, sales, market share and relative profits compared. Manage Decis Econ 30(3):141-153 
[23] Miller NH, Pazgal AI (2002) Relative performance as a strategic commitment mechanism. Manage Decis Econ 23(2):51-68

[24] Mira C, Gardini L, Barugola A, Cathala JC (1996) Chaotic Dynamics in Two-Dimensional Noninvertible Maps. World Scientific Singapore

[25] Panchuk A, Puu T (2009) Stability in a non-autonomous iterative system: an application to oligopoly. Comput Math Appl 58(10):2022-2034

[26] Puu T (1991) Chaos in duopoly pricing. Chaos Soliton Fract 1(6):573-581

[27] Ritz RA (2008) Strategic incentives for market share. Int J Ind Organ 26(2):586-597

[28] Singh N, Vives X (1984) Price and quantity competition in a differentiated duopoly. RAND J Econ 15(4):546-554

[29] Sklivas SD (1987) The strategic choice of managerial incentives. RAND J Econ 18(3):452-458

[30] Vickers J (1985) Delegation and the theory of the firm. Econ J 95:138-147 